“C2021 IEEE. Personal use of this material is permitted. Permission from IEEE must be obtained for all other uses, in any current or future media, including reprinting/republishing this material for advertising or promotional purposes, creating new collective works, for resale or redistribution to servers or lists, or reuse of any copyrighted component of this work in other works." 


\title{
EEG-based Brain-Computer Interfaces (BCls): A Survey of Recent Studies on Signal Sensing Technologies and Computational Intelligence Approaches and Their Applications
}

\author{
Xiaotong $\mathrm{Gu}^{\dagger}$, Zehong $\mathrm{Cao}^{\dagger}{ }^{*}$, Member, IEEE, Alireza Jolfaei, Member, IEEE, Peng Xu, Member, IEEE, \\ Dongrui Wu, Senior Member, IEEE, Tzyy-Ping Jung, Fellow, IEEE, and Chin-Teng Lin, Fellow, IEEE
}

\begin{abstract}
Brain-Computer interfaces (BCls) enhance the capability of human brain activities to interact with the environment. Recent advancements in technology and machine learning algorithms have increased interest in electroencephalographic (EEG)-based BCl applications. EEG-based intelligent $\mathrm{BCl}$ systems can facilitate continuous monitoring of fluctuations in human cognitive states under monotonous tasks, which is both beneficial for people in need of healthcare support and general researchers in different domain areas. In this review, we survey the recent literature on EEG signal sensing technologies and computational intelligence approaches in $\mathrm{BCl}$ applications, compensating for the gaps in the systematic summary of the past five years. Specifically, we first review the current status of $\mathrm{BCl}$ and signal sensing technologies for collecting reliable EEG signals. Then, we demonstrate state-of-the-art computational intelligence techniques, including fuzzy models and transfer learning in machine learning and deep learning algorithms, to detect, monitor, and maintain human cognitive states and task performance in prevalent applications. Finally, we present a couple of innovative $\mathrm{BCl}$-inspired healthcare applications and discuss future research directions in EEG-based $\mathrm{BCl}$ research.
\end{abstract}

\section{INTRODUCTION}

\subsection{An overview of brain-computer interfaces (BCls)}

\subsubsection{What is a $\mathrm{BCl}$ ?}

The first research papers on brain-computer interfaces (BCIs) were released in the 1970s. These works addressed an alternative transmission channel that does not depend on the normal peripheral nerve and muscle output paths of the brain [1]. The earliest concept of a BCI proposed measuring and decoding brainwave signals to control a prosthetic arm and carry out a desired action [2]. Later, a formal definition of the term 'BCI' was interpreted as a direct communication pathway between the human brain and an external device [3]. In the past decade, human BCIs have attracted substantial attention due to their extensive research potential.

The corresponding human BCI systems aim to translate human cognition patterns using brain activities. They use recorded brain activity to communicate with a computer to control external devices or environments in a manner that is compatible with the intentions of humans [4], such as controlling a wheelchair or robot as shown in Fig. 1 .

- X. Gu and Z. Cao are with the University of Tasmania, Australia.

- A. Jolfaei is with the Macquarie University, Australia.

- $P . X u$ is with the University of Electronic Science and Technology of China, China.

- D. Wu is with the Huazhong University of Science and Technology, China.

- T.P Jung is with the University of California, San Diego, USA.

- C.T. Lin is with the University of Technology Sydney, Australia. $\dagger$ Equal contribution.

* Corresponding author (Email address: zehong.cao@utas.edu.au)

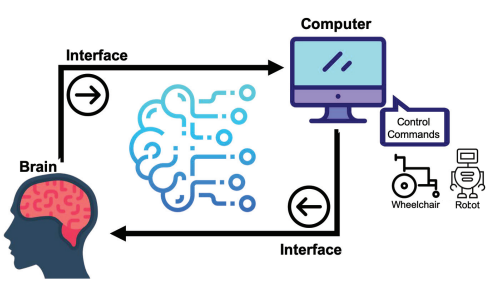

Fig. 1. Framework of a brain-computer interface $(\mathrm{BCl})$

There are two primary types of BCIs. The first type is active and reactive BCIs. An active BCI derives patterns from brain activity, which is directly and consciously controlled by the user independent of external events to control a device [5]. A reactive BCI extracts outputs from brain activities in reaction to external stimulation, which is indirectly modulated by the user to control an application. The second type is passive BCIs, which explore user's perception, awareness, and cognition without the purpose of voluntary control, resulting in an enriched human-computer interaction (HCI) with implicit information [6].

\subsubsection{Application areas}

The promising future of $\mathrm{BCIs}$ has encouraged the research community to interpret brain activities to establish various research directions for BCIs. General researchers from a wide range of areas of expertise, including computer science, data analysis, engineering, education, health, and psychology, can find related content and research trends relevant to their respective areas in this survey paper. 
Here, we address the best-known application areas in which BCIs have been widely explored and applied: (1) A BCI is recognised to offer the potential for an approach that uses intuitive and natural human mechanisms of cognitive processing to facilitate interactions [7]. Since the common methods of conventional HCI are mostly restricted to manual interfaces and the majority of other designs are not being extensively adopted [8], BCIs change how HCIs could be used in complex and demanding operational environments. BCIs could revolutionise mainstream HCIs for use in different areas such as computer-aided design (CAD) [9]. Using BCIs to monitor user states for intelligent assistive systems is also substantially conducted in entertainment and health areas [10]. (2) Another area where BCI applications are broadly used is as game controllers for entertainment. Some BCI devices are inexpensive, easily portable and easy to equip, which makes them feasible for broad use in entertainment communities. The compact and wireless BCI headsets developed for the gaming market are flexible, mobile, and require little effort to set up. Though their accuracy is not as precise as other BCI devices used in medical areas, they are still practical for game developers and have been successfully commercialised for the entertainment market. Some specific models [11] are combined with sensors to detect more signals such as facial expression, features from which can upgrade the usability for entertainment applications. (3) BCIs have also been fulfilling significant roles in neurocomputing for pattern recognition and machine learning based on brain signals and analysis of computational expert knowledge. Recently, studies have shown [12] [13] [14] that network neuroscience approaches have been used to quantify brain network reorganisation from different varieties of human learning. The results of these studies indicate optimisation of adaptive BCI architectures and the prospect of revealing the neural basis and future performance of BCI learning. (4) In the healthcare field, the brainwave headset, which can collect expressive information with the software development kit provided by the manufacturer, has been utilised to facilitate effective control of a robot by severely disabled people through subtle movements such as neck motion and blinking [15]. BCIs have also been used in assisting people who have lost the muscular capacity to restore communication and control over devices. A broadly investigated clinical area is focused on implementing BCI spelling devices. One well-known application of this domain is a P300based speller. By building upon the P300-based speller and using a BCI2000 platform [16] [17], the BCI speller achieves a positive result with respect to non-experienced users using this brain-controlled spelling tool. Another application area of $\mathrm{BCI}$ is the authentication process within the cybersecurity field [18], which is to utilise EEG-based systems to decide whether to reject or accept the claiming identity of a subject. The P300-BCI-based authentication system proposed by $\mathrm{Yu}$ et al. |19| showed the good potential of BCI for highly secured authentication systems.

Overall, BCIs have contributed to various fields of research. As briefly shown in Fig. 2, they are involved in game interaction entertainment, robot control, emotion recognition, fatigue detection, sleep quality assessment, and clinical fields, such as abnormal brain disease detection and prediction including applications to seizure, Parkinson's disease,

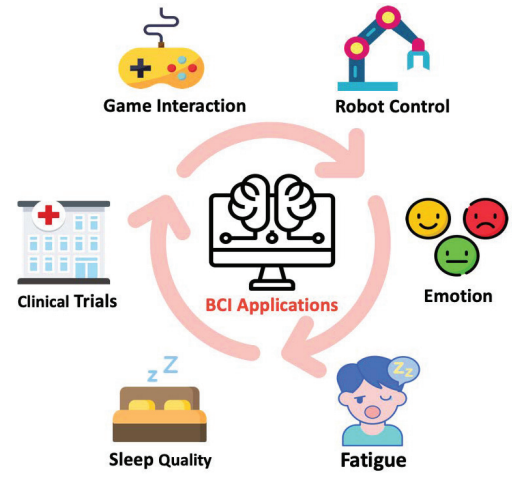

Fig. 2. $\mathrm{BCl}$ contributes to various fields of research

Alzheimer's disease, and schizophrenia.

\subsubsection{Brain imaging techniques}

Brain-sensing devices for BCIs can be categorised into three groups: invasive, partially invasive, and non-invasive [20]. In invasive and partially invasive devices, brain signals are collected from intracortical and electrocorticography (ECoG) electrodes with sensors tapping directly into the brain's cortex. As the invasive devices insert electrodes into the brain cortex, each electrode of the intracortical collection technique can provide spiking to produce the population's time developing an output pattern, which causes only a small sample of the complete set of neurons in bonded regions to be presented because microelectrodes can only detect spiking when they are in the proximity of a neuron. In this case, ECoG, as an extracortical invasive electrophysiological monitoring method, uses electrodes attached under the skull. With lower surgical risk, a rather high signal-tonoise ratio (SNR), and a higher spatial resolution compared with intracortical signals of invasive devices, ECoG offers superior potential in the medical area. In particular, ECoG has a wider bandwidth to gather significant information from functional brain areas to train a high-frequency BCI system, as well as high SNR signals that are less prone to artefacts arising from, for instance, muscle movements and eye blinks.

Even though reliable information of cortical and neuronal dynamics can be provided by invasive or partially invasive BCIs, when considering everyday applications, the potential benefit of increased signal quality is neutralised by the surgical risks and need for long-term implantation of invasive devices [21]. Recent studies focused on investigating the non-invasive technology, which uses external neuroimaging devices to record brain activity, including functional near-infrared spectroscopy (fNIRS), functional magnetic resonance imaging (fMRI), and electroencephalography (EEG). Specifically, fNIRS uses near-infrared (NIR) light to assess the aggregation level of oxygenated haemoglobin and deoxygenated haemoglobin. fNIRS depends on the haemodynamic response or blood-oxygen-level-dependent (BOLD) response to formulate functional neuroimages [22]. Because of the power limits of light and spatial resolution, fNIRS cannot be employed to measure cortical activity presented under $4 \mathrm{~cm}$ in the brain. Additionally, due to the fact that blood flow changes slower than electrical 
or magnetic signals, $\mathrm{Hb}$ and deoxy-Hb exhibit slow and steady variations, so the temporal resolution of fNIRS is comparatively lower than that of electrical or magnetic signals. fMRI monitors brain activities by assessing changes related to blood flow in brain areas, and it relies on the magnetic BOLD response, which enables fMRI to have a higher spatial resolution and collect brain information from deeper areas than fNIRS, since magnetic fields have better penetration than NIR light. However, similarly to fNIRS, the drawback of fMRI with low temporal resolutions is evident because of the blood flow speed constraint. With the merits of relying on the magnetic response, the fMRI technique also has another flaw since the magnetic fields are more prone to be distorted by deoxy- $\mathrm{Hb}$ than by $\mathrm{Hb}$ molecules. The most significant disadvantages of the use of fMRI in different scenarios are that it requires an expensive and heavy scanner to generate magnetic fields and that the scanner is not portable and requires substantial effort for movement.

\subsubsection{EEG-based BCls}

EEG signals, featuring direct measurements of cortical electrical activity and high temporal resolution, have been pursued extensively in many recent BCI studies [23] [24]. As the most generally used non-invasive technique, EEG electrodes can be installed in a headset device to collect EEG signals; this device is generally referred as an EEGbased BCI system. Considering the relative increases in signal quality, reliability and mobility compared with other imaging approaches, non-invasive EEG-based devices have been used as the most popular modality for real-world BCIs and clinical use [25].

EEG headsets can collect signals in several nonoverlapping frequency bands (e.g., Delta, Theta, Alpha, Beta, and Gamma). This is based on the powerful intra-band connection with distinct behavioural states [26], and the different frequency bands can present diverse corresponding characteristics and patterns. Furthermore, the temporal resolution is exceedingly high, reaching the millisecond level, and the risk for subjects is very low compared with invasive and other non-invasive techniques that require high-intensity magnetic field exposure. In this survey, we discussed different high portability and comparatively inexpensive EEG devices. A drawback of the EEG technique is that the signals have a low spatial resolution because of the limited number of electrodes; however, the temporal resolution is considerably high. When using EEG signals for BCI systems, the inferior SNR needs to be considered because objective factors such as environmental noise and subjective factors such as fatigue status might contaminate the EEG signals. The recent research conducted to cope with this disadvantage of EEG technology is also discussed in our survey.

A BCI makes it possible to observe a specific brain's response to specific stimuli events by recording small potential changes in EEG signals immediately after visual or audio stimuli appear. This phenomenon is formally called event-related potentials (ERPs), defined as slight voltages originating in the brain as responses to specific stimuli or events [27], which are then separated into visual evoked potentials (VEPs) and auditory evoked potentials (AEPs). For
EEG-based BCI studies, the P300 wave is a representative potential response of an ERP elicited in the brain cortex of a monitored subject, presenting as a positive deflection in voltage with a latency of roughly 250 to $500 \mathrm{~ms}$ [28]. In VEP tasks, rapid serial visual presentation (RSVP), the process of continuously presenting multiple images per second at high display rates, is considered to have potential in enhancing human-machine symbiosis [29]. Steady-State visual evoked potentials (SSVEPs) are a resonance phenomenon originating mainly in the visual cortex when a person is focusing visual attention on a light source flickering with a frequency above $4 \mathrm{~Hz}$ [30]. In addition, the psychomotor vigilance task (PVT) is a sustained-attention, reaction-timed task of measuring the speed with which subjects respond to a visual stimulus, and it correlates with the assessment of alertness, fatigue, or psychomotor skills [31].

\subsection{Our motivations and contributions}

\subsubsection{Motivations}

Recent (2015-2019) EEG survey articles focus primarily on separately summarising statistical features or patterns, collecting classification algorithms, or introducing deep learning models. For example, a recent survey [32] provided a comprehensive outline of the latest classification algorithms used in EEG-based BCIs, which include adaptive classifiers, transfer and deep learning, matrix and tensor classifiers, and several other miscellaneous classifiers. Although [32] believes that deep learning methods have not demonstrated convincing enhancement over some state-ofthe-art BCI methods, the results reviewed recently in [33] illustrated that some deep learning methods, for instance, convolutional neural networks (CNNs), generative adversarial networks (GANs), and deep belief networks (DBNs), have achieved outstanding performance in classification accuracy. However, there is no comparison drawn between deep learning neural networks and conventional machine learning methods to prove the improvement of modern neural network algorithms in EEG-based BCIs. Another recent survey [34] systematically reviewed articles published between 2010 and 2018 that applied deep learning to EEG in diverse domains by extracting and analysing various datasets to identify the research trend(s), but it did not include information about EEG sensors or hardware devices that collect EEG signals. Additionally, an up-to-date survey article released in early 2019 [35] reviewed brain signal categories for BCIs and deep learning techniques for BCI applications, with a discussion of applied areas for deeplearning-based BCIs. While this survey provided a systematic summary of relevant publications between 2015 and 2019, it did not thoroughly investigate machine learning, deep transfer learning, and fuzzy models that are used for non-stationary and non-linear EEG signal processing.

\subsubsection{Contributions}

The recent review articles we mentioned above lack a comprehensive survey of recent EEG sensing technologies, signal enhancement, relevant machine learning algorithms with transfer learning and fuzzy models, and deep learning methods for specific BCI applications, especially for healthcare systems. To develop and disseminate a BCI in real- 
world applications, we need to cover all of these technologies as opposed to focusing on signal processing or machine learning alone. In our survey, we aim to address all of these limitations and include the recently published BCI studies from 2015-2019. The main contributions of this study are summarised as follows:

- Advances in sensors and sensing technologies (Section 2).

- Characteristics of signal enhancement and online processing (Section 3).

- Recent machine learning algorithms of transfer learning and fuzzy models for BCI applications (Section 4).

- Recent deep learning algorithms and combined approaches for BCI applications (Section 5).

- Evolution of healthcare systems and applications in BCIs (Section 6).

In terms of the relationships among each contribution, we focus on EEG-based BCI research and discuss the pathway from signal processing to applications by first introducing the EEG signal and recent advancements in sensors and devices for non-invasive measurement (Section 2). Considering the importance of noise removal for EEG signal processing, we then review the recent studies on EEG signal enhancement and artefact handling technologies (Section 3). Regarding the recent development of feature extraction from processed EEG signals and analysis, we survey the recent studies employing machine learning (Section 4) and several well-recognised deep learning algorithms with diverse BCI applications (Section 5). Among all of these EEG-based BCI applications, we concentrate on reviewing the recent studies regarding the transformation of healthcare systems and $\mathrm{BCI}$ healthcare applications (Section 6), as we believe that these represent the most relevant sections and trends of EEGbased BCI research.

\section{Advances in Sensing Technologies}

\subsection{An overview of EEG sensors/devices}

Advanced sensor technology has enabled the development of smaller and smarter wearable EEG devices for lifestyle and related medical applications. In particular, recent advances in EEG monitoring technologies pave the way for wearable, wireless EEG monitoring devices with dry sensors. In this section, we summarise the advances in EEG devices with wet or dry sensors. We also list and compare some commercially available EEG devices, as shown in Fig. 3 and Table 1. with a focus on the number of channels, sampling rate, stationary or portable nature of the devices, and coverage of companies that can cater to the specific needs of EEG users.

\subsubsection{Wet sensor technology}

For non-invasive EEG measurements, wet electrode caps are attached to users' scalps with gels as the interface between sensors and scalps. The wet sensors relying on electrolytic gels provide a clean conductive path. The application of the gel interface is utilised to decrease the skin-electrode contact interface impedance, which could be uncomfortable and inconvenient for users and too time-consuming and laborious for everyday use [36]. Without the conductive gel, the electrode-skin impedance cannot be measured, and the quality of measured EEG signals could be compromised.

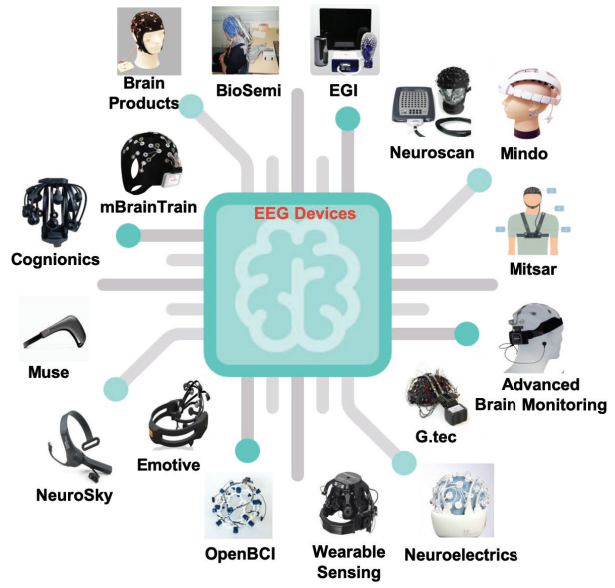

Fig. 3. Commercialised EEG devices for $\mathrm{BCl}$ applications

\subsubsection{Dry sensor technology}

Because the use of wet electrodes for collecting EEG data requires attaching sensors over the experimenter's skin, which is not desirable in real-world applications, the development of dry sensors of EEG devices has been dramatically enhanced over the past several years [37]. One of the major advantages of dry sensors, compared with wet counterparts, is that they substantially enhance system usability |38|; the headset is also very easy to wear and remove, which even allows skilled users to utilise it by themselves in a short period of time. For example, Siddharth et al. [39] designed biosensors to measure physiological activities to overcome the limitations of wet-electrode EEG equipment. These biosensors are dry-electrode based, and the signal quality is comparable to that obtained with wetelectrode systems, but without the need for skin abrasion or preparation or the use of gels. In a follow-up study [39], novel dry EEG sensors that could actively filter the EEG signal from ambient electrostatic noise were designed and evaluated with an ultra-low-noise and high-sampling analogue-to-digital converter module. The study compared the proposed sensors with commercially available EEG sensors (Cognionics Inc.) in an SSVEP BCI task, and the SSVEP detection accuracy was comparable between two sensors, with $74.23 \%$ average accuracy across all subjects.

Furthermore, regarding the trend of wearable biosensing devices, Chi et al. [40] [41] reviewed and designed wireless devices with dry and non-contact EEG electrode sensors. Chi et al. [41] developed a new integrated sensor controlling the sensitive input node to attain prominent input impedance, with complete shielding of the input node from the active transistor by bond pads, to the specially built chip package. Their experiment results, using data collected from a non-contact electrode on the top of the hair, demonstrated a maximum information transfer rate of $100 \%$ accuracy, thus indicating the promising future for dry and non-contact electrodes as viable tools for EEG applications and mobile BCIs.

The augmented $\mathrm{BCI}(\mathrm{ABCI})$ concept was proposed in [38] for everyday environments, with which signals are recorded via biosensors and processed in real-time to monitor human behaviour. An ABCI comprises non-intrusive 
and quick-setup EEG solutions, which require minimal or no training, to accurately collect long-term data with the benefits of comfort, stability, robustness, and longevity. In their study of a broad range of approaches to ABCIs, developing portable EEG devices using dry electrode sensors is a significant target for mobile human brain imaging, and future $\mathrm{ABCI}$ applications will be based on biosensing technology and devices.

\subsection{Commercialised EEG devices}

Table 1 lists 21 products of 17 brands along with eight attributes to provide a basic overview of EEG headsets. The attribute 'Wearable' indicates whether the monitored human subjects could wear the devices and move around without movement constraints, which partially depends on the transmission types and whether the headset devices are connected to software via wireless technologies such as WiFi or Bluetooth or via tethered connections. The numbers of channels of each EEG device can be categorised into three groups: a low-resolution group with 1 to 32 channels, a medium-resolution group with 33 to 128 channels, and a high-resolution group with more than 128 channels. Most brands offer more than one device, and therefore, the numbers of channels in Table 1 exhibit a wide range. The low-resolution devices mainly cover the frontal and temporal locations, some of which also deploy sensors to collect EEG signals from five locations, while the mediumand high-resolution groups can cover scalp locations more comprehensively. The numbers of channels also affect the EEG signal sampling rate of each device, with the lowand medium-resolution groups having a general sampling rate of $500 \mathrm{~Hz}$ and the high-resolution group achieving a sampling rate of higher than 1,000 Hz. Figure 3 presents all commercialised EEG devices listed in Table 1

\section{Signal Enhancement and Online Pro- CESSING}

\subsection{Artefact handling}

Based on a broad category of unsupervised learning algorithms for signal enhancement, blind source separation (BSS) estimates original sources and parameters of a mixing system and removes the artefact signals, such as eye blinks and movement, present in the sources [42]. There are several prevalent BSS algorithms in BCI research, including principal component analysis (PCA), canonical correlation analysis (CCA) and independent component analysis (ICA). PCA, one of the simplest BSS techniques, converts correlated variables to uncorrelated variables, named principal components (PCs), by the orthogonal transformation. However, the artefact components are usually correlated with EEG data, and the drift potentials are similar to EEG data, both of which would cause the PCA to fail to separate the artefacts [43]. CCA separates components from uncorrelated sources and detects a linear correlation between two multidimensional variables $|44|$ that have been applied in muscle artefact removal from EEG signals. In terms of ICA, it decomposes observed signals into the independent components (ICs) and reconstructs clean signals by removing the ICs that contain artefacts. ICA is the most utilised approach for artefact removal from EEG signals, so we review the methods that utilise ICA to support signal enhancement in the following sections.

\subsubsection{Eye blinks and movements}

Eye blinks are more prevalent when eyes are open, while rolling of the eyes might influence the eyes-closed condition. Additionally, the signals of eye movements located in the frontal area can affect further EEG analysis. To minimise the influence of eye contamination in EEG signals, visual inspection of artefacts and data augmentation approaches are often used to remove eye contamination.

Artefact subspace reconstruction (ASR) is an automatic component-based mechanism used as a pre-processing step to effectually remove large-amplitude or transient artefacts that contaminate EEG data. There are several limitations of ASR. ASR effectually removes artefacts from the EEG signals collected from a standard 20-channel EEG device, for which single-channel EEG recordings cannot be applied. Furthermore, without substantial cut-off parameters, the effectiveness of removing regularly occurring artefacts, such as eye blinks and eye movements, is limited. A possible enhancement by using an ICA-based artefact removal mechanism as a complement to ASR cleaning has been proposed [45]. A recent study in 2019 [46] also considered ICA and used an automatic IC classifier as a quantitative measure to separate brain signals and artefacts for signal enhancement. The above studies extended Infomax ICA [47], and the results showed that ASR removes more eye artefacts than brain components by using an optimal ASR parameter between 20 and 30 .

For online processing of EEG data in near real time to remove artefacts, a combination method of online ASR, online recursive ICA, and an IC classifier was proposed in [45] to remove large-amplitude transients as well as to compute, categorise, and remove artefactual ICs. For the eye movement-related artefacts, the results of their proposed methods have a fluctuating saccade-related IC EyeCatch score, and the altered version of EyeCatch in their study is still not an ideal method of eliminating eye-related artefacts.

\subsubsection{Muscle artefacts}

Contamination of EEG data by muscle activity is a wellrecognised and challenging problem. These artefacts can be generated by any muscle contraction or stretch in proximity to the recording sites, such as when the subject talks, sniffs, swallows, etc. The degree of muscle contraction or stretch will affect the amplitude and waveform of artefacts in EEG signals. Common techniques that have been used to remove muscle artefacts include regression methods, CCA, empirical mode decomposition (EMD), BSS, and EMD-BSS [43]. A combination of the ensemble EMD (EEMD) and CCA, named EEMD-CCA, was proposed to remove muscle artefacts by [48]. By testing on real-life, semi-simulated, and simulated datasets under single-channel, few-channel, and multichannel settings, the study results indicate that the EEMD-CCA method can effectively and accurately remove muscle artefacts, and it is an efficient signal processing and enhancement tool in healthcare EEG sensor networks. Other approaches are combining typical methods, such as using BSS-CCA followed by spectral-slope rejection to reduce 
TABLE 1

Overview of EEG Devices

\begin{tabular}{|c|c|c|c|c|c|c|c|c|}
\hline Brand & Product & Wearable & Sensor type & Channel no. & Locations & Sampling rate & Transmission & Weight \\
\hline NeuroSky & MindWave & Yes & Dry & 1 & $\mathrm{~F}$ & $500 \mathrm{~Hz}$ & Bluetooth & $90 \mathrm{~g}$ \\
\hline Emotiv & $\operatorname{EPOC}(+)$ & Yes & Dry & 5-14 & F, C, T, P,O & $500 \mathrm{~Hz}$ & Bluetooth & $125 \mathrm{~g}$ \\
\hline Muse & Muse 2 & Yes & Dry & $4-7$ & F, T & & Bluetooth & \\
\hline OpenBCI & EEG Electrode Cap Kit & Yes & Wet & 8- 21 & $\mathrm{~F}, \mathrm{C}, \mathrm{T}, \mathrm{P}, \mathrm{O}$ & & Cable & \\
\hline Wearable Sensing & DSI 24; NeuSenW & Yes & Wet; Dry & $7-21$ & $\mathrm{~F}, \mathrm{C}, \mathrm{T}, \mathrm{P}, \mathrm{O}$ & $300 / 600 \mathrm{~Hz}$ & Bluetooth & $600 \mathrm{~g}$ \\
\hline AIVI I veuro & eego mylab / eego sports & Yes & Dry & $32-256$ & $\mathrm{~F}, \mathrm{C}, \mathrm{T}, \mathrm{P}, \mathrm{O}$ & $\mathrm{Up}$ to $16 \mathrm{kHz}$ & $\mathrm{Wi}-\mathrm{Fi}$ & $500 \mathrm{~g}$ \\
\hline Neuroelectrics & STARSTIM: ENOBIO & Yes & Dry & $8-32$ & FC T P O & $125-500 \mathrm{~Hz}$ & Wi-Fi: USB & \\
\hline G.tec & g.NAUTILUS series & Yes & Dry & $8-64$ & $\mathrm{~F}, \mathrm{C}, \mathrm{T}, \mathrm{P}, \mathrm{O}$ & $500 \mathrm{~Hz}$ & Wireless & $140 \mathrm{~g}$ \\
\hline Advanced Brain Monitoring & B-Alert & Yes & Dry & $10-24$ & $\mathrm{~F}, \mathrm{C}, \mathrm{T}, \mathrm{P}, \mathrm{O}$ & $256 \mathrm{~Hz}$ & Bluetooth & $110 \mathrm{~g}$ \\
\hline Cognionics & Quick & Yes & Dry & $8-30 ;(64-128)$ & F, C, T, P, O & $250 \mathrm{~Hz} / 500 \mathrm{~Hz} / 1 \mathrm{kHz} / 2 \mathrm{kHz}$ & Bluetooth & $610 \mathrm{~g}$ \\
\hline mbrain Irain & Smarting & Yes & Wet & 24 & $\mathrm{~F}, \mathrm{C}, \mathrm{T}, \mathrm{P}, \mathrm{O}$ & $250-500 \mathrm{~Hz}$ & Bluetooth & $60 \mathrm{~g}$ \\
\hline Brain Products & LiveAmp & Yes & Dry & $8-64$ & $\mathrm{~F}, \mathrm{C}, \mathrm{T}, \mathrm{P}, \mathrm{O}$ & $250 \mathrm{~Hz} / 500 \mathrm{~Hz} / 1 \mathrm{kHz}$ & Wireless & $30 \mathrm{~g}$ \\
\hline Brain Products & AntiCHapmp & Yes & Dry & $32-160$ & $\mathrm{~F}, \mathrm{C}, \mathrm{T}, \mathrm{P}, \mathrm{O}$ & $10 \mathrm{kHz}$ & Wireless & $1.1 \mathrm{~kg}$ \\
\hline Biosemi & ActiveTwo & No & Wet (Gel) & 280 & F, C, T, P, O & $2 / 4 / 8 / 16 \mathrm{kHz}$ & Cable & $1.1 \mathrm{~kg}$ \\
\hline EGI & GES 400 & No & dry & $32-256$ & $\mathrm{~F}, \mathrm{C}, \mathrm{T}, \mathrm{P}, \mathrm{O}$ & $8 \mathrm{kHz}$ & Cable & \\
\hline Compumedics Neuroscan & Quick-Cap + Grael 4k & No & Wet & $32-256$ & $\mathrm{~F}, \mathrm{C}, \mathrm{T}, \mathrm{P}, \mathrm{O}$ & & Cable & \\
\hline Mitsar & Smart BCI EEG Headset & Yes & Wet & $24-32$ & $\mathrm{~F}, \mathrm{C}, \mathrm{T}, \mathrm{P}, \mathrm{O}$ & $2 \mathrm{kHz}$ & Bluetooth & $50 \mathrm{~g}$ \\
\hline Vilndo & Mindo series & Yes & Dry & $4-64$ & $\mathrm{~F}, \mathrm{C}, \mathrm{T}, \mathrm{P}, \mathrm{O}$ & & Wireless & \\
\hline
\end{tabular}

high-frequency muscle contamination $[49]$ and independent vector analysis (IVA) that takes advantage of both ICA and CCA by exploiting higher-order statistics (HOS) and second-order statistics (SOS) simultaneously to achieve high performance in removing muscle artefacts [50]. A more extensive survey on muscle artefact removal from EEG can be found in [43].

\subsubsection{Introducing a signal enhancement toolbox}

EEGLAB is one of the most widely used MATLAB toolboxes for processing of EEG and other electrophysiological data. It was developed by the Swartz Center for Computational Neuroscience and provides an interactive graphic user interface for users to apply ICA, time/frequency analysis (TFA) and standard averaging methods to the recorded brain signals. EEGLAB extensions, previously called EEGLAB plugins, are toolboxes that provide data processing and visualisation functions for the EEGLAB users to process the EEG data. At the time of writing, there are 106 extensions available on the EEGLAB website, with a broad functional range including data importing, artefact removal, and feature detection algorithms. Many extensions have been developed for artefact removal. For instance, the automatic artefact removal (AAR) toolbox is for automatic ocular and muscular artefact removal from EEG; cochlear implant artefact correction (CIAC), as its name implies, is an ICA-based tool particularly for correcting electrical artefacts arising from cochlear implants; the multiple artefact rejection algorithm (MARA) toolbox uses EEG features in the temporal, spectral and spatial domains to optimise a linear classifier to solve the component-reject vs. -accept problem and to remove loss electrodes; and the 'icablinkmetrics' toolbox aims at selecting and removing ICA components associated with eye blink artefacts using time-domain methods. Some of the toolboxes have more than one major function, such as artefact rejection and pre-processing: "clean_rawdata" is a suite of pre-processing methods including ASR for correcting and cleaning continuous data. ARfitStudio can be applied to quickly and intuitively correct event-related spiky artefacts as the first step of data pre-processing using "ARfit". ADJUST identifies and removes artefact-independent components automatically without affecting neural sources or data. A more comprehensive list of toolbox functions can be found on the EEGLAB website.

\subsection{EEG Online Processing}

For neuronal information processing and BCIs, the ability to monitor and analyse cortico-cortical interactions in real time is one of the trends in BCI research, along with the development of wearable BCI devices and effective approaches to remove artefacts. It is challenging to create a reliable real-time system that can collect, extract and pre-process dynamic data with artefact rejection and rapid computation. In the model proposed by [51], EEG data collected from a wearable, high-density (64-channel), and dry EEG device are first reconstructed by a 3,751-vertex mesh, anatomically constrained low resolution electrical tomographic analysis (LORETA), singular value decomposition (SVD)-based reformulation and automated anatomical labelling (AAL) before being forwarded to the Source Information Flow Toolbox (SIFT) and vector autoregressive (VAR) model. By applying regularised logistic regression and testing on both simulation and real data, their proposed system is capable of real-time EEG data analysis. Later, Mullen et al. [37] expanded their prior study by incorporating ASR for artefact removal; they implemented the anatomically constrained LORETA to localise sources and added the alternating direction method of multipliers and cognitive-state classification. The evaluation results of the proposed framework on simulated and real EEG data demonstrate the feasibility of the real-time neuronal system for cognitive state classification and identification. A subsequent study [52] aimed to present data for instantaneous incremental convergence. Online recursive least squares (RLS) whitening and an optimised online recursive ICA algorithm (ORICA) were validated for separating the blind sources from high-density EEG data. The experimental results prove the proposed algorithm's capability to detect non-stationary high-density EEG data and to extract artefact and principal brain sources quickly. The open-source Real-time EEG Source-mapping Toolbox (REST), which provides support for online artefact rejection and feature extraction, is available to inspire more real-time $\mathrm{BCI}$ research in different domain areas.

\section{Machine Learning and Fuzzy Models in BCI APPLICATIONS}

In this section, as shown in Fig. 4. we introduce two research areas, machine learning (ML) and fuzzy models, and review 


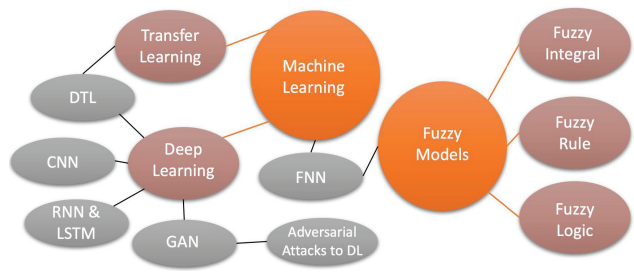

Fig. 4. Structure of Machine Learning, Deep Learning, and Fuzzy Models

the recent research outcomes of BCI applications. Machine learning is a subset of computational intelligence that comprises numerous research areas, including transfer learning (TL) and deep learning (DL). In Section 5, we introduce and survey some state-of-the-art architectures of deep learning neural networks, including CNNs, GANs, recurrent neural networks (RNNs), long short-term memory (LSTM), deep transfer learning (DTL), as well as their applications and recent researches in BCI. Fuzzy models, which apply fuzzy rules, fuzzy logic, and fuzzy measure theory (such as fuzzy integrals) to a fuzzy inference system (FIS), are preferable for processing non-linear and non-stationary EEG signals in BCI research. We also introduce fuzzy neural networks (FNNs), which are a combination of FIS and deep learning neural networks, and FNN BCI applications.

\subsection{An overview of machine learning}

Machine learning relies on general patterns of reasoning by computer systems to explore a specific task without providing explicit coded instructions. Machine learning tasks are generally classified into several types, including supervised learning and unsupervised learning [53]. In terms of supervised learning, it usually divides the data into two subsets during the learning process: a training set (a dataset to train a model) and a test set (a dataset to test the trained model). Supervised learning can be used for classification and regression tasks by applying what has been learned in the training stage using labelled examples to test the new data (testing data) in order to classify types of events or predict future events. In contrast, unsupervised machine learning is used when the data used for training are neither classified nor labelled. It involves only input data and refers to a probability function to describe a hidden structure, such as grouping or clustering of data points.

Performing conventional machine learning requires the creation of a model for training purposes. In EEG-based $\mathrm{BCI}$ applications, various types of models have been used and developed for machine learning. In the last ten years, the leading families of models used in BCIs include linear classifiers, neural networks, non-linear Bayesian classifiers, nearest neighbour classifiers, and classifier combinations [54]. The linear classifiers, such as linear discriminant analysis (LDA), regularised LDA, and support vector machine (SVM), classify discriminant EEG patterns using linear decision boundaries between feature vectors for each class. In the context of neural networks, they assemble layered human neurons to approximate any non-linear decision boundaries, where the most common type in BCI application is the multilayer perceptron (MLP) that typically uses

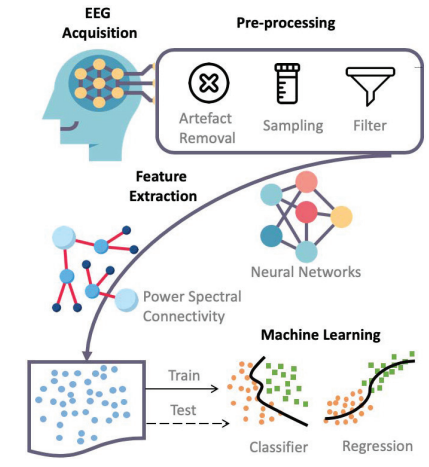

Fig. 5. Data pre-processing, pattern recognition and machine learning pipeline in BCls

only one or two hidden layers. Moving to a non-linear Bayesian classifier, such as a Bayesian quadratic classifier and a hidden Markov model (HMM), the probability distribution of each class is modelled, and Bayes rules are used to select the class to be assigned to the EEG patterns. Considering the physical distances of EEG patterns, nearest neighbour classifiers, such as the k nearest neighbour (kNN) algorithm, propose to assign a class to the EEG patterns based on the nearest neighbour. Finally, classifier combinations combine the outputs of multiple classifiers above or train them in a way that maximises their complementarity. The classifier combinations used for BCI applications can be enhanced, voting, or stacked combination approaches.

Additionally, to apply machine learning algorithms to EEG data, we need to pre-process EEG signals and extract features from the raw data, such as frequency band power features and connectivity features between two channels [55]. Fig. 5 demonstrates an EEG-based data pre-processing, pattern recognition and machine learning pipeline to extract features that represent EEG data in a compact and relevant way.

\subsection{Transfer learning}

\subsubsection{What is transfer learning}

Transfer learning is a set of methodologies to enhance the performance of a classifier initially trained on one task (also extended to one session or subject) based on information gained while learning another task. Transferring knowledge from the source domain to the target domain can act as a bias or a regulariser for solving the target task. Here, we provide a description of transfer learning based on the survey of Pan and Yang [56]. The source domain is known, and the target domain can be inductive (known) or transductive (unknown). Transfer learning is classified under three sub-settings in accord with the source and target tasks and domains: inductive transfer learning, transductive transfer learning, and unsupervised transfer learning.

In inductive transfer learning, available labelled data of the target domain are required, while the tasks of the source and target can be different regardless of their domains. Inductive transfer learning can be further categorised into two cases based on the availability of the labelled data. If data are available, a multitask learning method will be applied to the target and source tasks to learn the model 
simultaneously. Otherwise, a self-taught learning technique should be deployed. In terms of transductive transfer learning, the labelled data are available in the source domain instead of the target domain, while the target and source tasks are identical regardless of the domains (of the target and source). Transductive transfer learning can be further categorised into two cases based on whether the feature spaces between the source domain and target domain are the same. If they are the same, then the sample selection bias/covariance shift method should be applied. Otherwise, a domain adaptation technique should be deployed. Unsupervised transfer learning is applied if labelled data are not available in either the source or target domain while the target and source tasks are related but different. The target of unsupervised transfer learning is to resolve clustering, density estimation, and dimensionality reduction tasks in the target domain [57].

\subsubsection{Why do we need transfer learning}

One of the major hypotheses in conventional machine learning, such as the supervised learning techniques described above, is that the training data used to train the classifier and the test data used to evaluate the classifier belong to the same feature space and follow the same probability distribution. However, this hypothesis is often violated in many applications because of human variability [58]. For example, a change in the EEG data distribution typically occurs when data are acquired from different subjects or across sessions and time for the same subject. Additionally, since the EEG signals are variant and not static, extensive BCI sessions exhibit inconsistency in the classification performance [59].

Thus, transfer learning aims at coping with data that violate this hypothesis by exploiting knowledge acquired while learning a given task for solving a different but related task. The advances of transfer learning can relax the restrictions of BCIs since there is no need to calibrate from the beginning point, there is less noise for transferred information, and transfer learning relies on previously usable data to increase the size of datasets. All learning algorithms transfer knowledge to different tasks/domains, in which situation the skills should be transferred to enhance the performance and avoid a negative transfer.

\subsubsection{Where to transfer in $\mathrm{BCls}$}

In BCIs, discriminative and stationary information could be transferred across different domains. The selection of which types of information to transfer is based on the similarity between the target and source domains. If the domains are very similar and the data sample is small, then discriminative information should be transferred; if the domains are different while there could be common information across the target and source domains, then stationary information should be transferred to establish more invariable systems [60] [61].

Domain adaptation, a representative method of transductive transfer learning, attempts to find a strategy for transforming the data space in which the decision rules will classify all datasets. Covariate shifting is a technique that is highly related to domain adaptation, which is the most frequent situation encountered in BCIs. In covariate shifting, the input distributions of the training and test

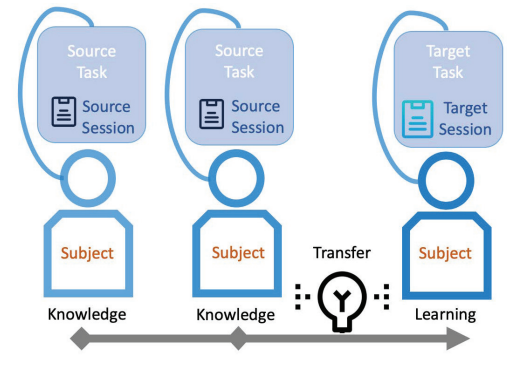

Fig. 6. Transfer learning in $\mathrm{BCls}$

samples are different, while the conditional distributions of output values are the same [62]. There exists an essential assumption: the marginal distribution of data changes from subjects (or sessions) to subjects (or sessions), and the decision rules for this marginal distribution remain unchanged. This assumption allows us to re-weight the training data from other subjects (or previous sessions) to correct the differences in the marginal distributions for the different subjects (or sessions).

Naturally, the effectiveness of transfer learning strongly depends on how closely the two circumstances are related. Transfer learning in BCI applications can be used to transfer information (a) from tasks to tasks, (b) from subjects to subjects, and (c) from sessions to sessions. As shown in Fig. 6. given a training dataset (e.g., source task, subject, or session), we attempt to find a transformation space in which training the model will improve the classification or prediction of samples from a new dataset (e.g., target task, subject, or session).

4.2.3.1 Transfer from tasks to tasks: In the BCI domain, where EEG signals are collected for analysis of subjects, the mental tasks and the operational tasks could be different but interdependent in some situations. For instance, in a laboratory environment, a mental task might be to assess a device action, such as mental subtraction or motor imagery (MI), while the operational task is the device action itself or the performance of the device action arising from ERPs. Transferring decision rules between different tasks would introduce novel signal variations and affect the error-related potential, which presents as a response recognised as an error by users [63]. The study of [64] showed that the signal variations originating from task-to-task transfer substantially influenced classification feature distribution and the classifier performance. Other results of their study are that the accuracy based on the baseline decreased when operational tasks and subtasks were generalised, while the differences in features were larger compared with non-error responses.

4.2.3.2 Transfer from subjects to subjects: For EEGbased BCIs, before applying features learned by the conventional approaches to different subjects, a training period of pilot data on each new subject is required due to intersubject variability |65|. In the driving drowsiness detection study of Wei et al. |66|, inter- and intra-subject variability were evaluated, and the feasibility of transferring models was validated by implementing hierarchical clustering in a large-scale EEG dataset collected from many subjects. The proposed subject-to-subject transfer framework comprises 
a large-scale model pool, which ensures that sufficient data are available for positive model transfer to obtain prominent decoding performance and small-scale baseline calibration data from the target subject as a selector of decoding models in the model pool. Without jeopardising the performance, their driving drowsiness detection results demonstrated $90 \%$ calibration time reduction.

In BCIs, cross-subject transfer learning, such as the leastsquares transformation (LST) method proposed by Chiang et al. [67], could be used to decrease the training data collection time. The experiments conducted to validate the LST method performance of cross-subject SSVEP data demonstrated the capability of reducing the number of training templates for an SSVEP BCI. Inter- and intra-subject transfer learning is also applied under unsupervised conditions when no labelled data are available. He and $\mathrm{Wu}[68]$ presented a method to align EEG trials directly in the Euclidean space across different subjects to increase the similarity. Their empirical results showed the potential of transfer learning from subjects to subjects in an unsupervised EEGbased BCI. In [69], He and Wu proposed a novel different-set domain adaptation approach for task-to-task and subject-tosubject transfer, which considers the very challenging case in which the source subject and the target subject have partially or completely different tasks. For example, the source subject may perform left-hand and right-hand MIs, whereas the target subject may perform feet and tongue MIs. They introduced a practical setting of different label sets for BCIs and proposed a novel label alignment (LA) approach to align the source label space with the target label space. LA only needs as few as one labelled sample from each class of the target subject, in which label alignment can be used as a pre-processing step before different feature extraction and classification algorithms and can be integrated with other domain adaptation approaches to achieve even better performance. For applying transfer learning in BCIs, especially EEG-based BCIs, subject-to-subject transfer among the same tasks is more frequently investigated.

For subject-to-subject transfer in single-trial ERP classification, $\mathrm{Wu}|70|$ proposed both online and offline weighted adaptation regularisation (wAR) algorithms to reduce the calibration effort. Experiments on a VEP oddball task and three different EEG headsets demonstrated that both online and offline wAR algorithms are effective. Wu also proposed a source domain selection approach, which selects the most beneficial source subjects for transfer. This approach can reduce the computational cost of wAR by approximately $50 \%$ without sacrificing the classification performance, thus making wAR more suitable for real-time applications.

Very recently, Cui et al. |71| proposed a novel approach, feature weighted episodic training (FWET), to completely eliminate the calibration requirement in subject-to-subject transfer in EEG-based driver drowsiness estimation. It integrates feature weighting to learn the importance of different features and episodic training for domain generalisation. FWET does not need any labelled or unlabelled calibration data from the new subject and hence could be very useful in plug-and-play BCIs.

4.2.3.3 Transfer from sessions to sessions: The assumption in session-to-session transfer learning in BCIs is that features extracted by the training module and algo- rithms can be applied to a different session of a subject for the same task. It is important to evaluate what is in common among training sections to optimise the decision distributions among different sessions.

Alamgir et al. |72| reviewed several transfer learning methodologies in BCIs that explore and utilise common training data structures of several sessions to reduce the training time and enhance the performance. Building on the comparison and analysis of other methods in the literature, Alamgir et al. proposed a general framework for transfer learning in BCIs, which is in contrast to a general transfer learning study that focuses on domain adaptation where individual session feature attributes are transferred. Their framework regards decision boundaries as random variables, so the distribution of decision boundaries could be obtained from previous sessions. With an altered regression method and the consideration of feature decomposition, their experiments on amyotrophic lateral sclerosis patients using an MI BCI revealed its effectiveness in learning structure. There are also some problematic conditions of the proposed transfer learning method, including the difficulty in balancing the initialisation of spatial weights and the necessity of adding an extra loop in the algorithm for determining the spectral and spatial combination.

In one of the latest paradigms studying imagined speech, in which a human subject imagines uttering a word without physical sound or movement, García-Salinas et al. |73| proposed a method to extract code words related to the EEG signals. After presenting a new imagined word along with the EEG signals of characteristic code words, the new word was merged with the prior class's histograms and a classifier for transfer learning. This study implies a general trend of applying session-based transfer learning to an imagined speech domain in EEG-based BCIs.

4.2.3.4 Transfer from headset to headset: Apart from the above cases of transfer learning in BCIs, ideally, a BCI system should be completely independent of any specific EEG headset such that the user can replace or upgrade his/her headset freely without re-calibration. This should greatly facilitate real-world applications of BCIs. However, this goal is very challenging to achieve. One step towards it is to use historical data from the same user to reduce the calibration effort for the new EEG headset.

$\mathrm{Wu}$ et al. [74] proposed active weighted adaptation regularisation (AwAR) for headset-to-headset transfer. It integrates wAR, which uses labelled data from the previous headset and handles class imbalance, and active learning, which selects the most informative samples from the new headset to label. Experiments on single-trial ERP classification showed that AwAR could significantly reduce the calibration data required for the new headset.

\subsection{Fuzzy Models}

\subsubsection{Why do we need fuzzy models?}

Currently, the classification processes of most machine learning methods are not easily interpretable or traceable. By comparison, fuzzy-rule based classification systems have developed sensible rules to process EEG activities based on our knowledge of neurophysiology and neuroscience, 


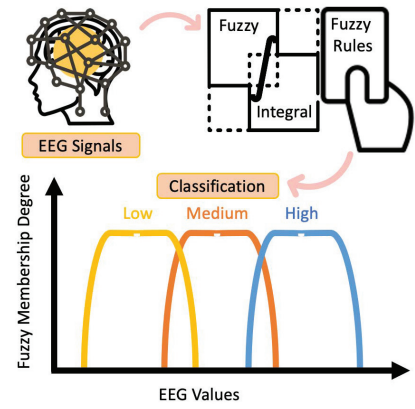

Fig. 7. Fuzzy sets, fuzzy rules, and fuzzy integrals for EEG signal classification

which are thus explainable. Additionally, conventional neural network classification methods, such as an MLP model, did not take into account the fact that EEG signals are non-linear and non-stationary. In comparison, a fuzzy set does not have the firm boundaries (the boundary transition is characterised by a membership function [75|) used for exploiting approximation techniques in neural networks, which makes neuro-fuzzy models preferable for extracting intrinsic EEG activities. Exploring these fuzzy models may be useful for understanding and improving BCIs learned automatically from EEG signals or possibly gaining new insights into BCIs. Here, we collect some fuzzy models from fuzzy sets and systems to estimate or accelerate BCI applications.

\subsubsection{EEG-based fuzzy models}

A fuzzy inference system (FIS) can be used in BCI applications to automatically extract fuzzy "If-Then" rules from the data that describe which input feature values correspond to which output category [76]. Such fuzzy rules confer the advantage of using flexible boundary conditions for BCI applications, EEG pattern classification, and interpreting what the FIS has learned, as shown in Fig. 7 Furthermore, fuzzy measure theory, such as a fuzzy integral, is suitable for applications where data fusion is required to consider possible data interactions [77], such as the fuzzy fusion of multiple information sources.

Fuzzy models integrated with neural networks have also been applied in BCI systems. For example, fuzzy neural networks (FNNs) combine the advantages of neural networks and FIS. This architecture is similar to that of neural networks, and the input (or weight) is fuzzified |78|. The FNN recognises the fuzzy rules and adjusts the membership function by tuning the connection weights. In particular, a self-organising neural fuzzy inference network (SONFIN) was proposed by [79] using a dynamic FNN architecture to create a self-adaptive architecture for the identification of the fuzzy model. The advantage of designing such a hybrid structure is more explanatory because it utilises the learning capability of the neural network.

Here, we summarise up-to-date fuzzy model solutions for EEG-based BCI systems and applications. By using fuzzy sets, Wu et al. [80] proposed to extend the multiclass EEG common spatial pattern (CSP) filters from classification to regression in a large-scale sustained-attention PVT and later further integrated them with Riemannian tangent space features for improved PVT reaction time estimation performance [81]. Considering the advantages of the fuzzy membership degree, [82] used a fuzzy membership function instead of a step function, which decreased the sensitivity of entropy values to noisy EEG signals. This improved the EEG complexity evaluation in resting state and SSVEP sessions [83], which are associated with healthcare applications [84].

By integrating fuzzy sets with EEG-based BCI domain adaptation, $\mathrm{Wu}$ et al. [85] proposed an online weighted adaptation regularisation for regression (OwARR) algorithm to reduce the amount of subject-specific calibration of the EEG data. Furthermore, by integrating fuzzy rules with domain adaptation, Chang et al. [86] generated a fuzzy rule-based brain-state-drift detector using Riemann-metricbased clustering, allowing the data distribution to be observable. By adopting fuzzy integrals [87], a motor-imagerybased $\mathrm{BCI}$ exhibited robust performance for offline singletrial classification and real-time control of a robotic arm. A follow-up work [88] explored a multimodel fuzzy fusionbased motor-imagery-based $\mathrm{BCI}$, which also considered the possible links between EEG patterns after employing the classification of conventional BCIs. Additionally, the fusion of multiple information sources is inspired by fuzzy integrals as well, such as fusing eye movement and EEG signals to enhance emotion recognition [89].

Moving to FNNs, due to the non-linear and nonstationary characteristics of EEG signals, the application of neural networks and fuzzy logic enables safe, accurate and reliable detection, as well as pattern identification. For example, a fuzzy neural detector was proposed for back-propagation with a fuzzy C-means algorithm [90] and Takagi-Sugeno fuzzy measurement [91] to identify the sleep stages. Furthermore, Liu et al. [90] proposed a recurrent selfevolving fuzzy neural network (RSEFNN) that employs an online gradient descent learning rule to predict EEG-based driving fatigue.

\section{Deep learning Algorithms With BCl Ap- PLICATIONS}

The representative architectures of deep learning include CNNs, GANs, RNNs, and LSTM models. Compared with machine learning technology, deep learning has been applied broadly in BCI applications, mainly because most current machine learning research has concentrated on static data: machine learning is not optimal for accurately categorising the rapidly changing brain signals [35]. In this section, we introduce spontaneous EEG applications with CNN architectures, the utilisation of GANs in recent research, and the procedures and applications of RNNs, especially LSTM models. We also illustrate DTL applied to deep learning algorithms and various transfer learning approaches, followed by a discussion of adversarial attacks on deep learning models for system testing.

\subsection{CNNs}

A CNN is a feed-forward neural network in which information flows unidirectionally from the input to the convolution operator and then to the output [92]. As shown in Fig. 8, a convolution operator includes at least three stacked layers in 


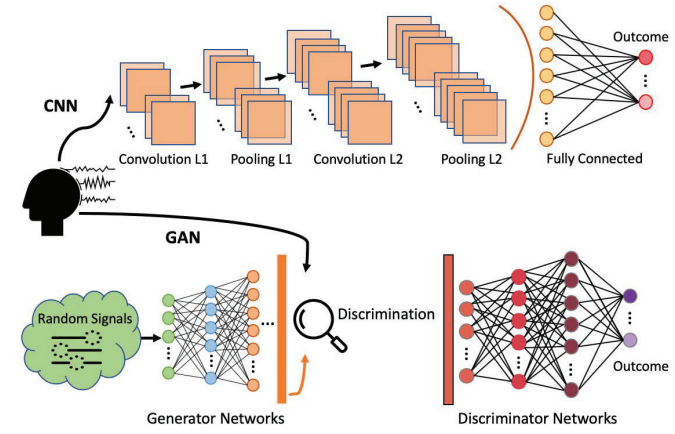

Fig. 8. CNNs and GANs for $\mathrm{BCl}$ applications

the CNN: a convolutional layer, a pooling layer, and a fully connected layer. The nature of CNNs with stacked layers is to reduce input data to easily identifiable formations with minimal loss, and distinctive spatial dependencies of EEG patterns can be captured by applying a CNN. For instance, a $\mathrm{CNN}$ has been used to automatically extract signal features from epileptic intracortical EEG data [93] and to perform automatic diagnoses to supersede the time-consuming procedure of expert visual examinations [94]. In addition to this, this review presents five recent $\mathrm{BCI}$ applications employing CNNs in fatigue, stress, sleep, MI, and emotional studies.

\subsubsection{Fatigue-related EEG}

As complex mental conditions, fatigue and drowsiness are states with a lack of vigilance that could lead to catastrophic incidents when subjects are conducting activities that require high and sustained attention, such as driving vehicles. Driving fatigue detection research has been attracting considerable attention in the BCI community [95] [96], especially in recent years with the significant advancement of CNNs in classification [97] [98]. An EEG-based spatialtemporal convolutional neural network (ESTCNN) was proposed by Gao et al. [99| for driver fatigue detection. This structure was applied to eight human subjects in an experiment in which multichannel EEG signals were collected. The framework comprises a core block that extracts temporal dependencies and combines them with dense layers for spatial-temporal EEG information processing and classification. It was illustrated that this method could consistently decrease the data dimension in the inference process and increase the reference response with computational efficiency. The results of the experiments with ESTCNN reached an accuracy rate of $97.37 \%$ in fatigue EEG signal classification. A CNN has also been used in other EEG-based fatigue recognition and evaluation applications. Yue and Wang [100] applied various fatigue levels' EEG signals to their multiscale CNN architecture, named "MorletInceptionNet," for visual fatigue evaluation. This framework uses a spacetime-frequency combined feature extraction strategy to extract raw features, after which multiscale temporal features are extracted by an inception architecture. The features are then provided to the $\mathrm{CNN}$ layers to classify visual fatigue evaluation. Their structure achieves better performance in classification accuracy than the other five state-of-the-art methodologies, which is proof of the effectiveness of CNNs in fatigue-related EEG signal processing and classification.

\subsubsection{Stress-related EEG}

Since stress is one of the leading causes of hazardous human behaviour and human errors that could cause dreadful industrial accidents, stress detection and recognition using EEG signals has become an important research area [101]. A recent study [102] proposed a new BCI framework with a CNN model and collected EEG signals from 10 construction workers whose cortisol levels, representing hormonerelated human stress, were measured to label task stress levels. The results of the proposed configuration obtained the maximum accuracy rate of $86.62 \%$. This study proved that the $\mathrm{BCI}$ framework with a $\mathrm{CNN}$ algorithm might be the ultimate classifier for EEG-based stress recognition.

\subsubsection{Sleep-related EEG}

Sleep quality is crucial for human health. Sleep stage classification, also called sleep scoring, has been investigated to understand, diagnose and treat sleep disorders [103]. Because of the lightness and portability of EEG devices, EEG is particularly suitable for recognising sleep scores. CNNs have been applied to sleep stage classification by numerous studies, and the approaches of CNNs using singlechannel EEG represent mainstream research investigations [94] [104], mainly due to simplicity [105]. The single-channel EEG-based method using a CNN for 5-class sleep stage conjecture in [103] exhibits competitive performance in sensible pattern detection and visualisation. The significance of this research for single-channel sleep EEG processing is that it does not require feature extraction from expert knowledge or signals to learn the most suitable features for the end-to-end classification task. Mousavi et al. [94] used a data-augmentation pre-processing method and applied raw EEG signals directly to nine convolutional layers and two fully connected layers without including feature extraction or feature selection. The simulation results of the study indicate an accuracy of over $93 \%$ for the classification of 2 to 6 sleep stage classes. Furthermore, a CNN-based combined classification and prediction framework, called multitask neural networks, was also considered for automatic sleep classification in a recent study [105]. It is stated that this framework has the ability to generate multiple decisions, reliability in forming a final decision by aggregation, and the capability to avoid the disadvantages of the conventional many-to-one approach.

\subsubsection{MI-related EEG}

MI refers to imagining the execution of the movement of a body part rather than conducting actual body movement in BCI systems [106]. MI is based on the fact that the brain activation will change and brain paths correlated to actually moving the body part will be activated. The CSP algorithm [107] is an effective spatial filter that searches for a discriminative subspace to simultaneously maximise one class variance and minimise the others to classify the movement actions. CNNs have also been employed for MI EEG data processing for stimulating the classification performance, and there is a stream of recent research trends of combining a CNN with CSP, improving the methodology, and enhancing the MI classification performance [108]. The MI classification framework proposed by Sakhavi, Guan 
and Yan [109] presents a new data temporal representation generated from the filter-bank CSP algorithm, with a CNN classification architecture. The accuracy on the 4-class MI $\mathrm{BCI}$ dataset demonstrates the usability and effectiveness of the proposed application. Olivas-Padilla and ChaconMurguia [110] presented two multiple MI classification methodologies that use a variation of the discriminative filter bank common spatial pattern (DFBCSP) to extract features, after which the outcome samples are input into a matrix that feeds one or multiple pre-optimised CNNs. It is stated that this proposed method could be an applicable alternative for multiple MI classification in a practical BCI application both online and offline.

\subsubsection{Emotion-related EEG}

Since it is believed that EEG signals contain comparatively comprehensive emotional information and better accessibility for affective research, while a CNN holds the capacity to take spatial information into account with two-dimensional filters, the CNN-based deep learning algorithm has been applied to EEG signals for emotion recognition and classification in numerous recent studies [111] [112] [113] [114]. Six basic emotional states that could be recognised and classified by using EEG signals [115] [116] include joy, sadness, surprise, anger, fear, and disgust, while the emotions could also be simply categorised in a binary classification as positive or negative [117]. To apply EEG signals to CNNbased modules, EEG signals could be directly introduced into the modules, or diverse entropy and power spectral density (PSD) features could be extracted as the input of the models. Three connectivity features extracted from EEG signals, the phase-locking value (PLV), Pearson correlation coefficient (PCC) and phase lag index (PLI), were examined in [117] with respect to the proposed three different CNN structures. A popular EEG-based emotion classification database, DEAP [118], was applied to the framework, and the PSD feature performance was enhanced by the connectivity features, with PLV matrices obtaining $99.72 \%$ accuracy when utilising CNN-5. Further, the dynamical graph CNN (DGCNN) has also been proposed for multichannel EEG emotion recognition. In the study of Song et al. [119], the presented DGCNN method uses a graph to model EEG features by learning intrinsic correlations between each EEG channel to produce an adjacency matrix, which is then applied to learn more discriminative features. The experiments conducted on the SJTU emotion EEG dataset (SEED) [120] and the DREAMER dataset [121] achieved recognition accuracy rates of $90.4 \%$ and $86.23 \%$, respectively.

\subsection{GANs}

\subsubsection{GANs for data augmentation}

In classification tasks, a substantial amount of real-world data is required for training machine learning and deep learning modules; in some cases, there are limitations in acquiring sufficient amounts of real data, or the investments of time and human resources could simply be too overwhelming. Proposed in 2014 and becoming more active in recent years, GANs are mainly used for data augmentation to address the question of how to generate artificial yet natural-looking samples to mimic real-world data via generative models so that the training data sample number can be increased [122].

A GAN includes two synchronously trained neural networks, a "generator network" and a "discriminator network," as shown in Fig. 8. The "generator" captures the input data distribution; its goal is to generate fake sample data, while the "discriminator" attempts to determine whether a sample originates from the real training data or is generated by the generator. These two neural networks aim to generate a set of samples created by the pre-trained generator and employ them in additional tasks, such as classification.

\subsubsection{EEG data augmentation}

The significance of applying a GAN for EEG is that it could address the major practical issue of insufficient training data. Abdelfattah, Abdelrahman and Wang [123] proposed a novel GAN model that learns statistical characteristics of the EEG signal and increases datasets to improve the classification performance. Their study showed that the method dramatically outperforms other generative models. The Wasserstein GAN with gradient penalty (WGAN-GP) proposed by Panwar et al. [124] incorporates a BCI classifier into the framework to synthesise EEG data and simulate time-series EEG data. WGAN-GP was applied to eventrelated classification and task classification with the classconditioned WGAN-CP. A GAN has also been used in EEG data augmentation for improving recognition performance, such as emotion recognition. The framework presented in [125] was built upon a conventional GAN, named the conditional Wasserstein GAN (CWGAN), to enhance EEGbased emotion recognition. The high-dimensional EEG data generated by the proposed GAN framework were evaluated by three indicators to ensure that high-quality synthetic data are appended to the manifold supplement. The positive experiment results on SEED and DEAP emotion recognition datasets proved the effectiveness of the CWGAN model. A conditional boundary equilibrium GAN-based EEG data augmentation method [126] for artificial differential entropy features generation was also proven to be effective in improving multimodal emotion recognition performance.

As a branch of deep learning, GANs have been employed to generate super-resolution image copies from low-resolution images. The GAN-based deep EEG superresolution method proposed by Corley and Huang [127] is a novel approach to generate high spatial resolution EEG data from low-resolution EEG samples by producing unsampled data from different channels. This framework can address the limitation of insufficient data collected from low-density EEG devices by interpolating multiple missing channels effectively.

To the best of our knowledge, in contrast to CNNs, GANs have been comparatively less studied in BCIs. One major reason for this is that the feasibility of using a GAN for generating time sequence data is yet to be fully evaluated [128]. In the investigation of the GAN performance in producing synthetic EEG signals, Fahimi et al. [128] used real EEG signals as random input to train a CNN-based GAN to produce synthetic EEG data and then evaluated the similarities in the frequency and time domains. Their results indicate that the EEG data generated from the GAN 
exhibit spatial, spectral, and temporal characteristics similar to those of real EEG signals. This initiates novel perspectives for future research regarding GANs in EEG-based BCIs.

\subsection{RNNs and LSTM}

As shown in Fig. 9, the recurrent procedure of an RNN describes a specific node $A$ in the time range $[1, t+1]$. At time $t$, node $A$ receives two input variables: $X_{t}$ denotes the input at time $t$ and the "backflow loop" represents the hidden state at time $[0, t-1]$. At time $t$, node $A$ exports the variable $h_{t}$. An LSTM network is a special kind of RNN that is capable of learning long-term dependencies. As shown in Fig. 9. an LSTM cell receives three inputs: the input $X$ at the current time $t$, the output $h$ of the previous time $t-1$, and the "input arrows" representing the hidden state of the previous time $t-1$. Then, the LSTM cell exports two outputs: the output $h$ and the hidden state (represented as the "out arrows ${ }^{\prime \prime}$ ) of the current time $t$.

Over the past several years, the research on the RNN framework in BCIs has increased substantially, with many studies showing that the results of the RNN-based methods outperform a benchmark or other conventional algorithms [129] and that combining an RNN with other deep neural networks such as a CNN can optimise the performance [130]. The RNN framework has also been applied to other EEG-based tasks, such as identification of individuals [26], hand motion identification [131], sleep staging [132], and emotion recognition [133]. Attia et al. [134] presented a hybrid architecture of a CNN and RNN model to categorise SSVEP signals in the time domain. In the research of applying an RNN to auditory stimulus classification, Moinnereau et al. [135] used a regulated RNN reservoir to classify three English vowels: $a, \mathrm{u}$ and $\mathrm{i}$. Their result showed an average accuracy rate of $83.2 \%$ with the RNN approach, which outperformed a deep neural network method. A framework aimed at addressing visual object classification was proposed by [136], which applied an RNN to EEG data invoked by visual stimulation. After discriminative brain activities were learned by the RNN, they were trained by a CNN-based regressor to project images onto the learned manifold. This automated object categorisation approach achieved an accuracy rate of approximately $83 \%$, which proved its comparability to those empowered merely by CNN models. It is worth noting that in [132], the best performing model among basic machine learning, CNNs, RNNs, and a combination of an RNN and a CNN (RCNN) is an RNN model with expert-defined features for sleep staging, which could inspire further research on combining the expert system with deep learning algorithms.

As a special type of RNN, LSTM has also been combined with CNN algorithms for a diverse range of EEG-based tasks. For automatic sleep stage scoring, Supratak et al. [137] employed a CNN to extract time-invariable features and an LSTM bidirectional algorithm for transitional rule learning. To predict human decisions from continuous EEG signals, Hasib et al. [138] proposed a hierarchical LSTM model with two layers encoding local-temporal correlations and temporal correlations to address non-stationarities of the EEG.

With the capabilities of learning sequential data and improving the classification performance, LSTM could also

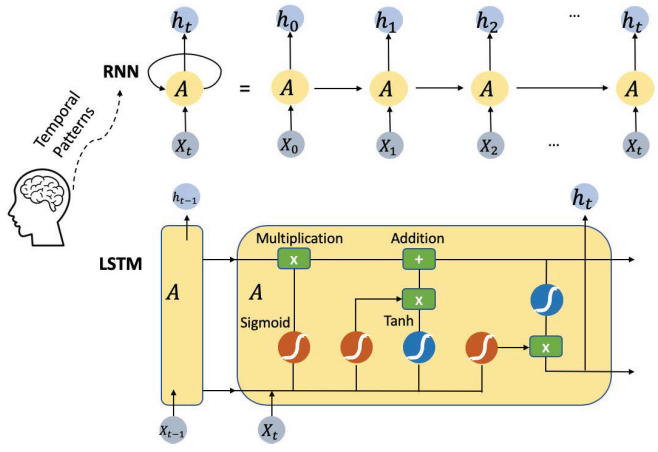

Fig. 9. Illustration of RNNs and LSTM

be added to other neural networks for temporal sequential pattern detection and optimisation of the overall prediction accuracy for the entire framework. For temporal sleep stage classification, Dong et al. [139] proposed a mixed neural network with LSTM with the capacity for learning a sleepscoring strategy automatically, in contrast to the decision tree approach in which rules are defined manually.

Because of the superior test-time performance of LSTMbased regression systems, LSTM models are also applied with GAN models to EEG signal analysis in different applications and domains, and some recent studies have exhibited their feasibility in EEG signal processing and preliminary results in EEG feature extraction. To build a mind-reading platform with EEG signals, Palazzo et al. [140] combined an LSTM-RNN model to extract visual class discriminative descriptors from EEG signals and a GAN model to read EEG signals from participants and convert those EEG descriptors into images. Their results showed that the generated images highly resemble those evoking the EEG signals processed by the LSTM model used for conditioning GANs. Krishna et al. [141] demonstrated a state-of-the-art automatic speech recognition model using an LSTM-based regression model and a GAN-based model to make a prediction by learning from EEG features.

\subsection{Deep Transfer Learning (DTL)}

Deep transfer learning (DTL) is the study of how to utilise knowledge from domain fields and effectively transfer knowledge by using deep neural networks. A recent survey [142] classified deep transfer learning into four categories: instance-based, mapping-based, network-based, and adversarial-based deep transfer learning. Specifically, the instance-based and mapping-based deep transfer learning approaches consider instances by adjusting weights from the source domain and mapping similarities from the source to the target domains, respectively. The main benefits of applying deep learning are reducing the time-consuming pre-processing while featuring engineering steps and capturing high-level symbolic features and imperceptible dependencies simultaneously [143]. The realisation of these two advantages is accomplished because deep learning operates directly on raw brain signals to learn identifiable information through back-propagation and deep structures, while transfer learning is commonly applied to improve the generalisation capacity for machine learning. 
Network-based deep transfer learning reuses the parts of the network pre-trained in the source domain, such as extracting the front-layers trained by a CNN. Adversarialbased deep transfer learning uses adversarial technology, such as a GAN, to find transferable features that are suitable for the two domains.

The combination of transfer learning and CNNs has been widely used in medical applications [144] [145] [146] and general application purposes for instance image classification [147] and object recognition [148]. In this section, we focus on transfer learning using a deep neural network and its EEG-based BCI applications.

MI EEG signal classification is one of the major areas where deep transfer learning is applied. Sakhavi and Guan [149| used a CNN model to transfer knowledge between subjects to decrease the calibration time for recording data and training the model. The EEG data pipeline of using a deep $\mathrm{CNN}$, transferring model parameters, finetuning on new data and using labels to regularise the finetuning/training process is a novel method for subject-tosubject and session-to-session deep transfer learning. Xu et al. [150] proposed a deep transfer $\mathrm{CNN}$ framework comprising a pre-trained VGG-16 CNN model and a target CNN model, between which the parameters are directly transferred, frozen and fine-tuned in the target model and MI dataset. The performance of their framework in terms of efficiency and accuracy exceeds many conventional methods such as the standard CNN and SVM. Dose et al. [151] applied a deep learning approach to an EEG-based MI BCI system in healthcare, aiming to enhance the present stroke rehabilitation process. The unified model they built includes $\mathrm{CNN}$ layers that learn generalised features and reduce the dimensionality and a conventional fully connected layer for classification. By using transfer learning in this approach for adapting global classifiers to single individuals and applying raw EEG signals to this model, the results of their study reached mean accuracy values of $86.49 \%, 79.25 \%$, and $68.51 \%$ for datasets with two, three and four classes, respectively. Regarding the effectiveness of alleviating the training burden with transfer learning, a recent research endeavour [152] encoded EEG features extracted from the conventional CSP by a separated channel CNN. The encoded features were then used to train a recognition network for MI classification. The accuracy of the proposed method outperformed multiple conventional machine learning algorithms.

\subsection{Adversarial Attacks on Deep Learning Models in $\mathrm{BCls}$}

Despite their outstanding performance, deep learning models are vulnerable to adversarial attacks, where deliberately designed small perturbations, which may be difficult to detect with human eyes or computer programs, are added to benign examples to mislead the deep learning model and cause dramatic performance degradation. This phenomenon was first discovered in 2014 in computer vision [153] and soon received great attention [154] [155] [156].

Adversarial attacks on EEG-based BCIs could also cause great damage. For example, EEG-based BCIs can be used to control wheelchairs or exoskeletons for the disabled [157], where adversarial attacks could cause malfunction.
In the worst case, adversarial attacks can harm users by intentionally driving them into danger. In clinical applications of BCIs in awareness/consciousness evaluation [157], adversarial attacks could lead to serious misdiagnosis.

Zhang's work [158] was the first to study adversarial attacks in EEG-based BCIs. They considered three different attack scenarios: 1) white-box attacks, where the attacker has access to all information of the target model, including its architecture and parameters; 2) black-box attacks, where the attacker can observe the target model's responses to inputs; and 3) grey-box attacks, where the attacker knows some but not all information about the target model, e.g., the training data on which the target model is tuned instead of its architecture and parameters. They showed that three popular CNN models in EEG-based BCIs, i.e., EEGNet [159], DeepCNN and ShallowCNN [160], can all be effectively attacked in all three scenarios.

Recently, Jiang et al. [161] showed that query synthesisbased active learning could help reduce the number of training EEG trials required in black-box adversarial attacks on the above three CNN classifiers, and Meng et al. [162] studied white-box target attacks for EEG-based BCI regression problems; i.e., by adding a tiny perturbation, they can change the estimated driver drowsiness level or user reaction time by at least a fixed amount. Liu et al. [163] proposed a novel total loss minimisation approach to generate universal adversarial perturbations for EEG classifiers. Their approach resolved two limitations of Zhang and Wu's approaches (the attacker needs to know the complete EEG trial in advance to compute the adversarial perturbation, and the perturbation needs to be computed specifically for each input EEG trial) and hence made adversarial attacks more practical.

\subsection{Comparison of experimental results for classifica- tion tasks}

Here, we compare the reviewed machine learning and deep learning algorithms in terms of feature extraction and experimental results for classification tasks based on some benchmark datasets in Table 2 Among these computational intelligence algorithms, deep learning models, especially the combination of different deep learning algorithms, generally outperformed the conventional machine learning (ML) approaches. However, deep learning models are sometimes referred to as black-box algorithms since there is no indication about how the classification decision is made [164], so the lack of transparency might impede future research on EEG-based BCIs.

The transfer learning method can directly align with EEG trials so that extracted patterns can be employed for the aligned trials without requiring any label information from new subjects [68]. The transfer learning method can also reduce the number of training templates [67] and conduct label alignment, which could achieve better performance when integrated with other feature extraction and classification algorithms [69]. The primary drawback of transfer learning is that it requires multiple tasks to support the experiment, and in general, the experimental outcomes might not be as good as those of deep learning algorithms on the same dataset. FNNs have better domain 
adaptation interpretability and signal processing capacity that are especially useful for handling non-linear and nonstationary EEG signals, but it is difficult to define the fuzzy membership or build multiple fuzzy systems.

Some other machine learning or deep learning approaches that achieved better classification accuracy but only utilised partial or private datasets for evaluation are not considered for comparisons in this survey.

\section{BCl-based Healthcare Systems}

Processed EEG signals are applied to diversified machine learning and deep learning algorithm architectures that can extract distinctive features for studies in diverse research domains. With the enhancement in affordability and quality of EEG headsets, EEG-based BCI research for classifying and predicting cognitive states has increased dramatically, such as tracking operators' inappropriate states for tasks and monitoring mental health and productivity [179]. EEG and other brain signals contain substantial information related to the health and disease conditions of the human brain. For instance, extraction of the "slowing down" features of EEG signals could be used to categorise neurodegenerative diseases [180].

One common brain disorder is epilepsy, for which patients suffer from recurrent unprovoked seizures. Clinically, EEG signals are among the leading indicators that can be monitored and studied for seizure electrical activity, while EEG-based BCI research can contribute to the prediction of epilepsy. In the medical area, EEG recordings are used for screening seizures of epilepsy patients with an automated seizure detection system. The gated recurrent unit RNN framework developed by [181] exhibited approximately $98 \%$ accuracy in epileptic seizure detection. Tsiouris et al. [182] introduced a two-layer LSTM network to assess seizure prediction performance by exploiting a broad scope of features before classification between EEG channels. The empirical results showed that the LSTM-based methodology outperformed conventional machine learning and CNNs in seizure prediction performance. Gupta et al. [183| proposed a novel method of EEG-based automatic seizure detection with a multirate filter bank structure and a statistical model to optimise signal attributes for better seizure classification accuracy. One of the main confusing elements for seizure detection is an artefact that appears in several EEG channels that could be misinterpreted due to wave and spike discharges similar to the occurrence of seizure. To optimise channel selection and accuracy for seizure detection with minimal false alarms, Shah et al. [184] proposed a CNNLSTM algorithm to reject artefacts and optimise the framework's performance for seizure detection. It is believed that the implementation of BCIs and real-time EEG signal processing are suitable for standard clinical application and caring for epilepsy patients [185].

Parkinson's disease (PD) is a progressive degenerative illness classified by degraded brain motor function, which, as an abnormal brain disease, is usually diagnosed with EEG signals. Oh et al. [186] proposed an EEG-based deep learning approach with a CNN architecture as a computeraided diagnosis system for PD detection, with positive performance result demonstrating its possibility in clinical usage. Ruffini et al. |187| presented a specific class of RNN frameworks called echo state networks (ESNs) to classify EEG signals collected from the random eye movement sleep behavioural disorder patients who eventually developed PD or Lewy Body Dementia and healthy control subjects. ESNs possessed the competence of RNNs in temporal pattern classification and could expedite training, and the test set performance accuracy of this ESNs model reached $85 \%$, but it did not exploit dynamical features in the input data streams.

Alzheimer's disease (AD) is one of the most mysterious pathologies and the causes of it are still insufficiently understood. BCIs with machine learning and deep learning models are also utilised in novel researches on classifying and detecting AD, while monitoring disease effects is increasingly significant for clinical intervention. For supporting clinical investigations, EEG signal screening of people who are vulnerable to AD could be utilised to discover the origination of $\mathrm{AD}$ development. With the potential for classification in a CNN, Morabito et al. [188] proposed a deep learning model with multiple convolutional subsampling layers and attained an average $80 \%$ accuracy in categorising sets of EEG data from two different classifications of subjects: mild cognitive impairment subjects, a prodromal version of $\mathrm{AD}$, and a healthy control group of the same age. Simpraga et al. [189| used machine learning with multiple EEG biomarkers to enhance the AD classification performance and demonstrated the effectiveness of their research in improving the disease identification accuracy and support in clinical trials.

Comparable to using deep learning models with multiple EEG biomarkers for AD classification, machine learning techniques have also been applied to EEG biomarkers for diagnosing schizophrenia. Shim et al. |190| used sensor and source level EEG features to classify schizophrenia patients and healthy control subjects. The result of their research indicated that the proposed tool could be promising in supporting schizophrenia diagnosis. Chu et al. [191] presented a modified deep learning architecture with a voting layer for individual schizophrenia classification of EEG streams. The high classification accuracy results in their study indicated the framework's feasibility in categorising firstepisode schizophrenia patients and healthy control subjects.

As a non-conventional neurorehabilitation methodology, BCIs have been investigated for assisting and aiding motor impairment rehabilitation, such as for patients who suffered and survived stroke, which is a common disease that generally results in patient's impaired mobility [192]. Non-invasive BCIs, such as EEG-based applications, support volitional transmission of brain signals to aid hand movements. BCIs offer great potential in facilitating motor impairment rehabilitation via the utilisation of assistive sensation by rewarding cortical action related to sensory-motor features [193]. Frolov et al. [194] investigated the effectiveness of rehabilitation for stroke survivors with BCI training sessions, and the research results of the participating patients indicated that combining BCIs with physical therapy could enhance the results of post-stroke motor impairment rehabilitation. Other researchers also found that using BCIs for motor impairment rehabilitation of post-stroke patients could help them regain bodily functions and improve their 
TABLE 2

Comparison of experimental results in classification tasks

\begin{tabular}{|c|c|c|c|}
\hline EEG Dataset & Algorithm Category & Method & Average Performance \\
\hline \multicolumn{4}{|l|}{ Motor-Imagery } \\
\hline BCI2000 MI Dataset ${ }^{1}$ & $\begin{array}{l}\text { Conventional ML } \\
\text { FNN } \\
\text { CNN; TL } \\
\text { CNN+LSTM } \\
\text { RNN } \\
\text { CNN+RNN }\end{array}$ & $\begin{array}{c}\text { Distributionally robust semi-supervised learning [165] } \\
\text { Discrete wavelet transform (DWT) and TSK (Tagaki-Sugeno-Kang) FNN 166] } \\
\text { CNN; TL 151] } \\
\text { CNN; 1D-convolutional_LSTM 167] } \\
\text { MTLEEG [168] } \\
\text { Spatio-Temporal representations-CNN-RNN 169] }\end{array}$ & $\begin{array}{c}77.01 \% \\
79.4 \% \\
80.38 \% ; 86.49 \% \\
99.58 \% \\
97.86 \% \\
98.3 \%\end{array}$ \\
\hline BCI Competition III dataset $3 \mathrm{a}^{2}$ & $\begin{array}{l}\text { Conventional ML } \\
\text { FNN } \\
\text { CNN }\end{array}$ & $\begin{array}{c}\text { Multiclass support matrix machine (MSMM) } \\
\text { RoCSP-SRIT2NFIS (self-regulated interval type-2 neuro-fuzzy inference system) 171] } \\
\text { CNN 108] }\end{array}$ & $\begin{array}{c}88 \% \\
92.94 \% \\
93.75 \%\end{array}$ \\
\hline BCI Competition IV dataset $2 \mathrm{a}^{3}$ & $\begin{array}{l}\text { Conventional ML } \\
\text { TL } \\
\text { FNN } \\
\text { RNN } \\
\text { CNN }\end{array}$ & $\begin{array}{c}\text { MSMM } \\
\text { Euclidean space data alignment (EA)-CSP-LDA 68] } \\
\text { RoCSP-SRIT2NFIS [171] } \\
\text { Gated recurrent unit (GRU)-KIV N 172] } \\
\text { CNN 110] }\end{array}$ & $\begin{array}{l}64.8 \% \\
73.53 \% \\
83.26 \% \\
73.56 \% \\
80.03 \%\end{array}$ \\
\hline \multicolumn{4}{|l|}{ Emotion-Recognition } \\
\hline DEAP $^{4}$ & $\begin{array}{l}\text { TL } \\
\text { Fuzzy-ML } \\
\text { GAN } \\
\text { CNN } \\
\text { LSTM }\end{array}$ & $\begin{array}{c}\text { Maximum independence domain adaptation (MIDA) 173] 174] } \\
\text { Fuzzy C-mean (FCFCM) [175] } \\
\text { Wasserstein generative adversarial network domain adaptation (WGANDA) 176] } \\
\text { CNN } 112] \\
\text { LSTM-RNIN 177] }\end{array}$ & $\begin{array}{c}48.93 \% \\
55.96 \%(\mathrm{a}), 55.77 \%(\mathrm{v}), 60.94 \%(\mathrm{~d}){ }^{*} \\
66.85 \%(\mathrm{a}), 67.99 \%(\mathrm{v}){ }^{*} \\
83.88 \% \\
85.65 \% \text { (a), } 85.45 \%(\mathrm{v}), 87.99 \%(\mathrm{l})\end{array}$ \\
\hline SEED $^{5}$ & $\begin{array}{l}\text { TL } \\
\text { TL } \\
\text { GAN } \\
\text { RNN }\end{array}$ & $\begin{array}{c}\text { Maximum independence domain adaptation (MIDA) }[173] \\
\text { Subspace alignment auto-encoder (SAAE) classification } \\
\text { 178] } \\
\text { Wasserstein generative adversarial network domain adaptation (WGANDA) 176] } \\
\text { Spatial-temporal recurrent neural network (STRNN) [133] }\end{array}$ & $\begin{array}{l}72.47 \% \\
81.81 \% \\
87.07 \% \\
89.5 \%\end{array}$ \\
\hline
\end{tabular}

${ }^{4}$ http://www.eecs.qmul.ac.uk/mmv/datasets/deap/ ${ }^{5}$ http://bcmi.sjtu.edu.cn/ seed/seed.html ${ }^{*}$ a: arousal; v: valence; d: dominance; l: liking

life quality [195] [196] [197].

BCIs have also been employed in other healthcare areas such as investigation of migraine, pain and depressive disorders [198] [199] [200| [201] [202]. Patil et al. [203] proposed an artificial neural network with supervised classifiers for EEG classification to detect migraine subjects. Their positive results confirmed that the EEG-based neural network classification framework could be used for migraine detection and as a substitution for migraine diagnosis. Cao et al. [204] [84 presented a multiscale relative inherent fuzzy entropy application for SSVEP-EEG signals of two migraine phases: the pre-ictal phase before migraine attacks and the interictal phase, which is the baseline. Their study found that for migraine patients, compared with healthy control subjects, there are changes in the EEG complexity in a repetitive SSVEP environment. Their study proved that inherent fuzzy entropy could be used in visual stimulus environments for migraine studies and offers potential in pre-ictal migraine prediction. EEG signals have also been monitored and analysed to prove the correlation between cerebral cortex spectral patterns and chronic pain intensity [205]. BCI-based signal processing approaches can also be used in training for phantom limb pain control by helping patients reorganise the sensorimotor cortex with the practice of hand control [206]. The potential of BCIs in healthcare for the general public may attract more novel research in the near future.

Machine learning and deep neural networks have been productively applied to EEG signals for screening, recognition and diagnosis of various neurological disorders, and recent researches revealed some important findings for depression detection with BCIs [207] [208]. Li et al. [208] proposed an EEG-based CAD system with a CNN architecture and the transfer learning method, indicating the critical temporal information of EEG to significantly improve the accuracy for the framework. Liao et al. [209] proved in their research that the 8 electrodes of EEG devices from the temporal areas can provide higher accuracy in major depression detection compared with other scalp areas. Acharya et al. [207] proposed a CNN approach for depression screening experiments on EEG signals of depression patients and healthy control subjects. Their study achieved accuracy rates of $93.5 \%$ and $96.0 \%$ for left and right hemisphere EEG signals, and confirmed the findings of a theory that depression is linked to a hyperactive right hemisphere, which could inspire future BCIs researches on depression detection and diagnosis.

\section{Discussion}

In this review, we highlighted the recent studies in the field of EEG-based BCI research by analysing approximately 200 papers published between 2015 and 2019 regarding developing signal sensing technologies and applying computational intelligence approaches to EEG data. Although the advances of dry sensors, wearable devices, toolboxes for signal enhancement, transfer learning, fuzzy models, and deep learning have improved the performance of EEGbased BCI systems, real-world usability challenges still remain, such as the prediction or classification performance and robustness in complex BCI scenarios.

In terms of sensing technology, the evolution of dry sensors is attributed to the cost-effectiveness and availability of EEG devices, which in turn stimulates more researches in developing enhanced sensors. The current trend in sensor techniques focuses on augmenting signal quality by improving sensors and emphasising user experience when collecting signals via BCI devices. Fiedler et al. [210] presented a basis for improved EEG cap designs of dry multipin electrodes in their research on polymer-based multichannel EEG electrode systems. Their study focused on the correlation of EEG recording quality with applied force and resulting contact pressure. Considering the comfort of wearing an EEG device, Lin et al. [211] developed a soft and pliable pad for an augmented wire-embedded and silicon-based dry-contact sensor to ensure scalp contact on hair-covered sites and exhibited good performance and feasibility for applications. Chen et al. [48] proposed flexible-material-based 
wearable sensors for monitoring EEG and other biosignals with applications in smart personal devices and e-health.

Furthermore, we believe that the following computational intelligence approaches and their applications will be significant for EEG-based BCI studies in the near future: (1) Closed-loop (CL) BCI: A CL BCI method detects human cognitive states to establish a closed-loop interaction between brain responses and musical or visual stimuli, such as healthcare electrical stimulation therapies [212] and stable controls using reinforcement learning [213]. (2) Adaptive EEG-based BCI: Based on the illustration of transfer learning and DTL, the benefits of transferring extracted features and training models among diverse subjects or tasks are apparent, such as improving the training efficiency and enhancing the classification accuracy. Therefore, it would be encouraging to pursue experiments with adaptive EEG-based BCI training. (3) Hybrid BCI: A hybrid $\mathrm{BCI}$ system comprises two or more BCIs or integrates at least one BCI with other technical or physiological signals, which can be a future focus of research for fusing multiple outcomes to improve classification accuracy [214] [215]. (4) Augmented reality (AR) linkage BCI: The scientific community is also investigating the conjunction of technology and interfaces for HCIs, e.g., the combination of AR and EEG-based BCIs [216]. Previous research integrated a popular protocol used in exogenous BCIs, SSVEPs, with visual stimuli rendering on an AR headmounted display (HMD), such as the smart glasses used in [217], and assessed the SSVEP responses by measuring EEG signals while the users performed tasks [218] [219]. With the accessibility of AR HMDs and commercialised non-invasive $\mathrm{BCI}$ devices, this augmentation becomes feasible and effective in developing integrated AR-BCI devices. (5) Adversarial attacks in BCI: Current EEG-based BCIs are vulnerable to adversarial attacks if EEG signals are contaminated by fake or noisy information generated from deep learning models, such as a GAN, so there is an urgent need to develop strategies to defend against such attacks.

\section{Conclusion}

In this paper, we systematically surveyed the recent advances in dry sensors, wearable devices, signal enhancement, machine learning algorithms including fuzzy models and transfer learning, and deep learning neural networks. We also reviewed healthcare applications of EEG-based BCIs and noted open challenges and future directions. Various types of computational intelligence approaches enable us to learn increasingly reliable neural features and understand human knowledge from EEG signals. In addition to the complementary contributions of this survey, we covered the development of EEG-based BCI studies in a diverse range of domains. We hope that researchers from the disciplines of computer science, data analysis, engineering, education, health, and psychology will find the contents and research trends in this survey relevant to their research areas in order to extend EEG-based BCI research directions.

\section{ACKNOWLEDGEMENT}

This work was supported in part by grants from the University of Tasmania and the Australian Research Council (ARC) under discovery grant DP180100670 and DP180100656. This work was also supported in part by grants from US NSF (CBET-1935860, NCS-1734883, IP-1719130, and SMA1540943) and the US Army Research Lab STRONG Program to TPJ. In addition, we credit the authors for the icons made from www.flaticon.com.

\section{REFERENCES}

[1] J. J. Vidal, "Toward direct brain-computer communication," Annual review of Biophysics and Bioengineering, vol. 2, no. 1, pp. 157180, 1973.

[2] C. Guger, W. Harkam, C. Hertnaes, and G. Pfurtscheller, "Prosthetic control by an eeg-based brain-computer interface (bci)," in Proc. aaate 5th european conference for the advancement of assistive technology. Citeseer, 1999, pp. 3-6.

[3] J. Wolpaw and E. W. Wolpaw, Brain-computer interfaces: principles and practice. OUP USA, 2012.

[4] F. Lotte, L. Bougrain, and M. Clerc, "Electroencephalography (eeg)-based brain-computer interfaces," Wiley Encyclopedia of Electrical and Electronics Engineering, pp. 1-20, 1999.

[5] E. E. Fetz, "Real-time control of a robotic arm by neuronal ensembles," Nature neuroscience, vol. 2, no. 7, p. 583, 1999.

[6] T. O. Zander and C. Kothe, "Towards passive brain-computer interfaces: applying brain-computer interface technology to human-machine systems in general," Journal of neural engineering, vol. 8, no. 2, p. 025005, 2011.

[7] S. S. Shankar and R. Rai, "Human factors study on the usage of bci headset for 3d cad modeling," Computer-Aided Design, vol. 54, pp. 51-55, 2014.

[8] B. Kerous, F. Skola, and F. Liarokapis, "Eeg-based bci and video games: a progress report," Virtual Reality, vol. 22, no. 2, pp. 119135, 2018.

[9] R. Rai and A. V. Deshpande, "Fragmentary shape recognition: A bci study," Computer-Aided Design, vol. 71, pp. 51-64, 2016.

[10] M. Ienca, J. Fabrice, B. Elger, M. Caon, A. S. Pappagallo, R. W. Kressig, and T. Wangmo, "Intelligent assistive technology for alzheimer's disease and other dementias: a systematic review," Journal of Alzheimer's Disease, vol. 56, no. 4, pp. 1301-1340, 2017.

[11] T. McMahan, I. Parberry, and T. D. Parsons, "Modality specific assessment of video game player's experience using the emotiv," Entertainment Computing, vol. 7, pp. 1-6, 2015.

[12] N. A. Badcock, K. A. Preece, B. de Wit, K. Glenn, N. Fieder, J. Thie, and G. McArthur, "Validation of the emotiv epoc eeg system for research quality auditory event-related potentials in children," Peer], vol. 3, p. e907, 2015.

[13] K. Finc, K. Bonna, M. Lewandowska, T. Wolak, J. Nikadon, J. Dreszer, W. Duch, and S. Kühn, "Transition of the functional brain network related to increasing cognitive demands," Human brain mapping, vol. 38, no. 7, pp. 3659-3674, 2017.

[14] C. Schmidt, D. Piper, B. Pester, A. Mierau, and H. Witte, "Tracking the reorganization of module structure in time-varying weighted brain functional connectivity networks," International journal of neural systems, vol. 28, no. 04, p. 1750051, 2018.

[15] A. Siswoyo, Z. Arief, and I. A. Sulistijono, "Application of artificial neural networks in modeling direction wheelchairs using neurosky mindset mobile (eeg) device," EMITTER International Journal of Engineering Technology, vol. 5, no. 1, pp. 170-191, 2017.

[16] F. D. V. Fallani and D. S. Bassett, "Network neuroscience for optimizing brain-computer interfaces," Physics of life reviews, 2019.

[17] W. A. Jang, S. M. Lee, and D. H. Lee, "Development bci for individuals with severely disability using emotiv eeg headset and robot," in 2014 International Winter Workshop on Brain-Computer Interface (BCI). IEEE, 2014, pp. 1-3.

[18] A. Jolfaei, X.-W. Wu, and V. Muthukkumarasamy, "On the feasibility and performance of pass-thought authentication systems," in 2013 Fourth International Conference on Emerging Security Technologies. IEEE, 2013, pp. 33-38.

[19] M. Yu, N. Kaongoen, and S. Jo, "P300-bci-based authentication system," in 2016 4th International Winter Conference on BrainComputer Interface (BCI). IEEE, 2016, pp. 1-4.

[20] A. Girouard, E. T. Solovey, L. M. Hirshfield, K. Chauncey, A. Sassaroli, S. Fantini, and R. J. Jacob, "Distinguishing difficulty levels with non-invasive brain activity measurements," in IFIP Conference on Human-Computer Interaction. Springer, 2009, pp. $440-452$. 
[21] F. Velasco-Álvarez, S. Sancha-Ros, E. García-Garaluz, Á. Fernández-Rodríguez, M. T. Medina-Juliá, and R. RonAngevin, "Uma-bci speller: an easily configurable p300 speller tool for end users," Computer methods and programs in biomedicine, vol. 172, pp. 127-138, 2019.

[22] T. Arichi, G. Fagiolo, M. Varela, A. Melendez-Calderon, A. Allievi, N. Merchant, N. Tusor, S. J. Counsell, E. Burdet, C. F. Beckmann et al., "Development of bold signal hemodynamic responses in the human brain," Neuroimage, vol. 63, no. 2, pp. 663-673, 2012.

[23] R. A. Ramadan and A. V. Vasilakos, "Brain computer interface: control signals review," Neurocomputing, vol. 223, pp. 26-44, 2017.

[24] P. Aricò, G. Borghini, G. Di Flumeri, N. Sciaraffa, and F. Babiloni, "Passive bci beyond the lab: current trends and future directions," Physiological measurement, vol. 39, no. 8, p. 08TR02, 2018.

[25] G. Schalk, D. J. McFarland, T. Hinterberger, N. Birbaumer, and J. R. Wolpaw, "Bci2000: a general-purpose brain-computer interface (bci) system," IEEE Transactions on biomedical engineering, vol. 51, no. 6, pp. 1034-1043, 2004.

[26] X. Zhang, L. Yao, C. Huang, T. Gu, Z. Yang, and Y. Liu, "Deepkey: An eeg and gait based dual-authentication system," arXiv preprint arXiv:1706.01606, 2017.

[27] S. Sur and V. Sinha, "Event-related potential: An overview," Industrial psychiatry journal, vol. 18, no. 1, p. 70, 2009.

[28] H. Serby, E. Yom-Tov, and G. F. Inbar, "An improved p300-based brain-computer interface," IEEE Transactions on neural systems and rehabilitation engineering, vol. 13, no. 1, pp. 89-98, 2005.

[29] S. Lees, N. Dayan, H. Cecotti, P. Mccullagh, L. Maguire, F. Lotte, and D. Coyle, "A review of rapid serial visual presentation-based brain-computer interfaces," Journal of neural engineering, vol. 15, no. 2, p. 021001, 2018.

[30] M. Nakanishi, Y. Wang, X. Chen, Y.-T. Wang, X. Gao, and T.P. Jung, "Enhancing detection of ssveps for a high-speed brain speller using task-related component analysis," IEEE Transactions on Biomedical Engineering, vol. 65, no. 1, pp. 104-112, 2017.

[31] Y. Li, A. Vgontzas, I. Kritikou, J. Fernandez-Mendoza, M. Basta, S. Pejovic, J. Gaines, and E. O. Bixler, "Psychomotor vigilance test and its association with daytime sleepiness and inflammation in sleep apnea: clinical implications," Journal of clinical sleep medicine, vol. 13, no. 09, pp. 1049-1056, 2017.

[32] F. Lotte, L. Bougrain, A. Cichocki, M. Clerc, M. Congedo, A. Rakotomamonjy, and F. Yger, "A review of classification algorithms for eeg-based brain-computer interfaces: a 10 year update," Journal of neural engineering, vol. 15, no. 3, p. 031005, 2018.

[33] A. Craik, Y. He, and J. L. Contreras-Vidal, “Deep learning for electroencephalogram (eeg) classification tasks: a review," Journal of neural engineering, vol. 16, no. 3, p. 031001, 2019.

[34] Y. Roy, H. Banville, I. Albuquerque, A. Gramfort, T. H. Falk, and J. Faubert, "Deep learning-based electroencephalography analysis: a systematic review," Journal of neural engineering, 2019.

[35] X. Zhang, L. Yao, X. Wang, J. Monaghan, and D. Mcalpine, “A survey on deep learning based brain computer interface: Recent advances and new frontiers," arXiv preprint arXiv:1905.04149, 2019.

[36] T. C. Ferree, P. Luu, G. S. Russell, and D. M. Tucker, "Scalp electrode impedance, infection risk, and eeg data quality," Clinical Neurophysiology, vol. 112, no. 3, pp. 536-544, 2001.

[37] T. R. Mullen, C. A. Kothe, Y. M. Chi, A. Ojeda, T. Kerth, S. Makeig, T.-P. Jung, and G. Cauwenberghs, "Real-time neuroimaging and cognitive monitoring using wearable dry eeg," IEEE Transactions on Biomedical Engineering, vol. 62, no. 11, pp. 2553-2567, 2015.

[38] L.-D. Liao, C.-T. Lin, K. McDowell, A. E. Wickenden, K. Gramann, T.-P. Jung, L.-W. Ko, and J.-Y. Chang, "Biosensor technologies for augmented brain-computer interfaces in the next decades," Proceedings of the IEEE, vol. 100, no. Special Centennial Issue, pp. 1553-1566, 2012.

[39] Siddharth, A. N. Patel, T. Jung, and T. J. Sejnowski, "A wearable multi-modal bio-sensing system towards real-world applications," IEEE Transactions on Biomedical Engineering, vol. 66, no. 4, pp. 1137-1147, April 2019.

[40] Y. M. Chi, T.-P. Jung, and G. Cauwenberghs, "Dry-contact and noncontact biopotential electrodes: Methodological review," IEEE reviews in biomedical engineering, vol. 3, pp. 106-119, 2010.

[41] Y. M. Chi and G. Cauwenberghs, "Wireless non-contact eeg/ecg electrodes for body sensor networks," in 2010 International Conference on Body Sensor Networks. IEEE, 2010, pp. 297-301.

[42] K. T. Sweeney, T. E. Ward, and S. F. McLoone, "Artifact removal in physiological signals-practices and possibilities," IEEE transactions on information technology in biomedicine, vol. 16, no. 3, pp. 488-500, 2012.

[43] X. Jiang, G.-B. Bian, and Z. Tian, "Removal of artifacts from eeg signals: a review," Sensors, vol. 19, no. 5, p. 987, 2019.

[44] L. Dong, Y. Zhang, R. Zhang, X. Zhang, D. Gong, P. A. ValdesSosa, P. Xu, C. Luo, and D. Yao, "Characterizing nonlinear relationships in functional imaging data using eigenspace maximal information canonical correlation analysis (emicca)," NeuroImage, vol. 109, pp. 388-401, 2015.

[45] L. Pion-Tonachini, S.-H. Hsu, C.-Y. Chang, T.-P. Jung, and S. Makeig, "Online automatic artifact rejection using the realtime eeg source-mapping toolbox (rest)," in 2018 40th Annual International Conference of the IEEE Engineering in Medicine and Biology Society (EMBC). IEEE, 2018, pp. 106-109.

[46] C.-Y. Chang, S.-H. Hsu, L. Pion-Tonachini, and T.-P. Jung, “Evaluation of artifact subspace reconstruction for automatic artifact components removal in multi-channel eeg recordings," IEEE Transactions on Biomedical Engineering, 2019.

[47] T.-W. Lee, M. Girolami, and T. J. Sejnowski, "Independent component analysis using an extended infomax algorithm for mixed subgaussian and supergaussian sources," Neural computation, vol. 11, no. 2, pp. 417-441, 1999.

[48] X. Chen, Q. Chen, Y. Zhang, and Z. J. Wang, "A novel eemd-cca approach to removing muscle artifacts for pervasive eeg," IEEE Sensors Journal, 2018.

[49] A. S. Janani, T. S. Grummett, T. W. Lewis, S. P. Fitzgibbon, E. M Whitham, D. DelosAngeles, H. Bakhshayesh, J. O. Willoughby, and K. J. Pope, "Improved artefact removal from eeg using canonical correlation analysis and spectral slope," Journal of neuroscience methods, vol. 298, pp. 1-15, 2018.

[50] X. Chen, H. Peng, F. Yu, and K. Wang, "Independent vector analysis applied to remove muscle artifacts in eeg data," IEEE Transactions on Instrumentation and Measurement, vol. 66, no. 7, pp. 1770-1779, 2017.

[51] T. Mullen, C. Kothe, Y. M. Chi, A. Ojeda, T. Kerth, S. Makeig G. Cauwenberghs, and T.-P. Jung, "Real-time modeling and 3d visualization of source dynamics and connectivity using wearable eeg," in 2013 35th annual international conference of the IEEE engineering in medicine and biology society (EMBC). IEEE, 2013, pp. 2184-2187.

[52] S.-H. Hsu, T. R. Mullen, T.-P. Jung, and G. Cauwenberghs, "Realtime adaptive eeg source separation using online recursive independent component analysis," IEEE transactions on neural systems and rehabilitation engineering, vol. 24, no. 3, pp. 309-319, 2015.

[53] N. Kasabov, "Evolving fuzzy neural networks for supervised/unsupervised online knowledge-based learning," IEEE Transactions on Systems, Man, and Cybernetics, Part B (Cybernetics), vol. 31, no. 6, pp. 902-918, 2001.

[54] S. B. Kotsiantis, I. D. Zaharakis, and P. E. Pintelas, "Machine learning: a review of classification and combining techniques," Artificial Intelligence Review, vol. 26, no. 3, pp. 159-190, 2006.

[55] I. Daly, S. J. Nasuto, and K. Warwick, "Brain computer interface control via functional connectivity dynamics," Pattern recognition, vol. 45, no. 6, pp. 2123-2136, 2012.

[56] S. J. Pan and Q. Yang, "A survey on transfer learning," IEEE Transactions on knowledge and data engineering, vol. 22, no. 10, pp. 1345-1359, 2009.

[57] Z. Wang, Y. Song, and C. Zhang, "Transferred dimensionality reduction," in Joint European Conference on Machine Learning and Knowledge Discovery in Databases. Springer, 2008, pp. 550-565.

[58] A. M. Azab, J. Toth, L. S. Mihaylova, and M. Arvaneh, "A review on transfer learning approaches in brain-computer interface," in Signal Processing and Machine Learning for Brain-Machine Interfaces. Institution of Engineering and Technology, 2018, pp. 81-101.

[59] H. A. Abbass, J. Tang, R. Amin, M. Ellejmi, and S. Kirby, “Augmented cognition using real-time eeg-based adaptive strategies for air traffic control," in Proceedings of the human factors and ergonomics society annual meeting, vol. 58, no. 1. SAGE Publications Sage CA: Los Angeles, CA, 2014, pp. 230-234.

[60] W. Samek, F. C. Meinecke, and K.-R. Müller, "Transferring subspaces between subjects in brain-computer interfacing," IEEE Transactions on Biomedical Engineering, vol. 60, no. 8, pp. 22892298, 2013. 
[61] P. Wang, J. Lu, B. Zhang, and Z. Tang, "A review on transfer learning for brain-computer interface classification," in 2015 5th International Conference on Information Science and Technology (ICIST). IEEE, 2015, pp. 315-322.

[62] H. Shimodaira, "Improving predictive inference under covariate shift by weighting the log-likelihood function," Journal of statistical planning and inference, vol. 90, no. 2, pp. 227-244, 2000.

[63] R. Chavarriaga, A. Sobolewski, and J. d. R. Millán, "Errare machinale est: the use of error-related potentials in brain-machine interfaces," Frontiers in neuroscience, vol. 8, p. 208, 2014.

[64] I. Iturrate, L. Montesano, and J. Minguez, "Task-dependent signal variations in eeg error-related potentials for brain-computer interfaces," Journal of neural engineering, vol. 10, no. 2, p. 026024, 2013.

[65] X. Chen, Y. Wang, M. Nakanishi, X. Gao, T.-P. Jung, and S. Gao, "High-speed spelling with a noninvasive brain-computer interface," Proceedings of the national academy of sciences, vol. 112, no. 44, pp. E6058-E6067, 2015.

[66] C.-S. Wei, Y.-P. Lin, Y.-T. Wang, C.-T. Lin, and T.-P. Jung, "A subject-transfer framework for obviating inter-and intra-subject variability in eeg-based drowsiness detection," NeuroImage, vol. 174, pp. 407-419, 2018.

[67] K.-J. Chiang, C.-S. Wei, M. Nakanishi, and T.-P. Jung, "Crosssubject transfer learning improves the practicality of real-world applications of brain-computer interfaces," in 2019 9th International IEEE/EMBS Conference on Neural Engineering (NER). IEEE, 2019, pp. 424-427.

[68] H. He and D. Wu, "Transfer learning for brain-computer interfaces: A euclidean space data alignment approach," IEEE Transactions on Biomedical Engineering, vol. 67, no. 2, pp. 399-410, 2020.

[69] —_ "Different set domain adaptation for brain-computer interfaces: A label alignment approach," IEEE Transactions on Neural Systems and Rehabilitation Engineering, vol. 28, no. 5, pp. 10911108,2020

[70] D. Wu, "Online and offline domain adaptation for reducing bci calibration effort," IEEE Transactions on human-machine Systems, vol. 47, no. 4, pp. 550-563, 2016.

[71] Y. Cui, Y. Xu, and D. Wu, "Eeg-based driver drowsiness estimation using feature weighted episodic training," IEEE transactions on neural systems and rehabilitation engineering, vol. 27, no. 11, pp. 2263-2273, 2019.

[72] V. Jayaram, M. Alamgir, Y. Altun, B. Scholkopf, and M. GrosseWentrup, "Transfer learning in brain-computer interfaces," IEEE Computational Intelligence Magazine, vol. 11, no. 1, pp. 20-31, 2016.

[73] J. S. García-Salinas, L. Villaseñor-Pineda, C. A. Reyes-García, and A. A. Torres-García, "Transfer learning in imagined speech eegbased bcis," Biomedical Signal Processing and Control, vol. 50, pp. 151-157, 2019

[74] D. Wu, V. J. Lawhern, W. D. Hairston, and B. J. Lance, "Switching eeg headsets made easy: Reducing offline calibration effort using active weighted adaptation regularization," IEEE Transactions on Neural Systems and Rehabilitation Engineering, vol. 24, no. 11, pp. 1125-1137, 2016.

[75] J.-S. R. Jang, C.-T. Sun, and E. Mizutani, "Neuro-fuzzy and soft computing-a computational approach to learning and machine intelligence [book review]," IEEE Transactions on automatic control, vol. 42, no. 10, pp. 1482-1484, 1997.

[76] L. Fabien, L. Anatole, L. Fabrice, and A. Bruno, "Studying the use of fuzzy inference systems for motor imagery classification," IEEE transactions on neural systems and rehabilitation engineering, vol. 15, no. 2, pp. 322-324, 2007.

[77] M. Sugeno, "Fuzzy measures and fuzzy integrals-a survey," in Readings in Fuzzy Sets for Intelligent Systems. Elsevier, 1993, pp. 251-257.

[78] J. J. Buckley and Y. Hayashi, "Fuzzy neural networks: A survey," Fuzzy sets and systems, vol. 66, no. 1, pp. 1-13, 1994.

[79] C.-F. Juang and C.-T. Lin, "A recurrent self-organizing neural fuzzy inference network," IEEE Transactions on Neural Networks, vol. 10, no. 4, pp. 828-845, 1999.

[80] D. Wu, J.-T. King, C.-H. Chuang, C.-T. Lin, and T.-P. Jung, “Spatial filtering for eeg-based regression problems in brain-computer interface (bci)," IEEE Transactions on Fuzzy Systems, vol. 26, no. 2, pp. 771-781, 2017.

[81] D. Wu, B. J. Lance, V. J. Lawhern, S. Gordon, T.-P. Jung, and C.-T. Lin, "Eeg-based user reaction time estimation using riemannian geometry features," IEEE Transactions on Neural Systems and Rehabilitation Engineering, vol. 25, no. 11, pp. 2157-2168, 2017.

[82] Z. Cao and C.-T. Lin, "Inherent fuzzy entropy for the improvement of eeg complexity evaluation," IEEE Transactions on Fuzzy Systems, vol. 26, no. 2, pp. 1032-1035, 2017.

[83] Z. Cao, W. Ding, Y.-K. Wang, F. K. Hussain, A. Al-Jumaily, and C.-T. Lin, "Effects of repetitive ssveps on eeg complexity using multiscale inherent fuzzy entropy," Neurocomputing, 2019.

[84] Z. Cao, C.-T. Lin, K.-L. Lai, L.-W. Ko, J.-T. King, K.-K. Liao, J.-L. Fuh, and S.-J. Wang, "Extraction of ssveps-based inherent fuzzy entropy using a wearable headband eeg in migraine patients," IEEE Transactions on Fuzzy Systems, 2019.

[85] D. Wu, V. J. Lawhern, S. Gordon, B. J. Lance, and C.-T. Lin, "Driver drowsiness estimation from eeg signals using online weighted adaptation regularization for regression (owarr)," IEEE Transactions on Fuzzy Systems, vol. 25, no. 6, pp. 1522-1535, 2016.

[86] Y.-C. Chang, Y.-K. Wang, D. Wu, and C.-T. Lin, "Generating a fuzzy rule-based brain-state-drift detector by riemann-metricbased clustering," in 2017 IEEE International Conference on Systems, Man, and Cybernetics (SMC). IEEE, 2017, pp. 1220-1225.

[87] S.-L. Wu, Y.-T. Liu, T.-Y. Hsieh, Y.-Y. Lin, C.-Y. Chen, C.-H. Chuang, and C.-T. Lin, "Fuzzy integral with particle swarm optimization for a motor-imagery-based brain-computer interface," IEEE Transactions on Fuzzy Systems, vol. 25, no. 1, pp. 21-28, 2016.

[88] L.-W. Ko, Y.-C. Lu, H. Bustince, Y.-C. Chang, Y. Chang, J. Ferandez, Y.-K. Wang, J. A. Sanz, G. P. Dimuro, and C.-T. Lin, "Multimodal fuzzy fusion for enhancing the motor-imagery-based brain computer interface," IEEE Computational Intelligence Magazine, vol. 14, no. 1, pp. 96-106, 2019.

[89] Y. Lu, W.-L. Zheng, B. Li, and B.-L. Lu, “Combining eye movements and eeg to enhance emotion recognition," in Twenty-Fourth International Joint Conference on Artificial Intelligence, 2015.

[90] Y.-T. Liu, Y.-Y. Lin, S.-L. Wu, C.-H. Chuang, and C.-T. Lin, "Brain dynamics in predicting driving fatigue using a recurrent self-evolving fuzzy neural network," IEEE transactions on neural networks and learning systems, vol. 27, no. 2, pp. 347-360, 2015.

[91] T.-H. Tsai, L.-J. Kau, and K.-M. Chao, "A takagi-sugeno fuzzy neural network-based algorithm with single-channel eeg signal for the discrimination between light and deep sleep stages," in 2016 IEEE Biomedical Circuits and Systems Conference (BioCAS). IEEE, 2016, pp. 532-535.

[92] H. Cecotti and A. Graser, "Convolutional neural networks for p300 detection with application to brain-computer interfaces," IEEE transactions on pattern analysis and machine intelligence, vol. 33, no. 3, pp. 433-445, 2010.

[93] A. Antoniades, L. Spyrou, C. C. Took, and S. Sanei, "Deep learning for epileptic intracranial eeg data," in 2016 IEEE 26th International Workshop on Machine Learning for Signal Processing (MLSP). IEEE, 2016, pp. 1-6.

[94] Z. Mousavi, T. Y. Rezaii, S. Sheykhivand, A. Farzamnia, and S. Razavi, "Deep convolutional neural network for classification of sleep stages from single-channel eeg signals," Journal of neuroscience methods, p. 108312, 2019.

[95] C.-H. Chuang, Z. Cao, J.-T. King, B.-S. Wu, Y.-K. Wang, and C.-T. Lin, "Brain electrodynamic and hemodynamic signatures against fatigue during driving," Frontiers in neuroscience, vol. 12, p. 181, 2018.

[96] Z. Cao, C.-H. Chuang, J.-K. King, and C.-T. Lin, “Multi-channel eeg recordings during a sustained-attention driving task," Scientific data, vol. 6, 2019.

[97] H. Zeng, C. Yang, G. Dai, F. Qin, J. Zhang, and W. Kong, "Eeg classification of driver mental states by deep learning," Cognitive neurodynamics, vol. 12, no. 6, pp. 597-606, 2018.

[98] E. J. Cheng, K.-Y. Young, and C.-T. Lin, “Image-based eeg signal processing for driving fatigue prediction," in 2018 International Automatic Control Conference (CACS). IEEE, 2018, pp. 1-5.

[99] Z. Gao, X. Wang, Y. Yang, C. Mu, Q. Cai, W. Dang, and S. Zuo, "Eeg-based spatio-temporal convolutional neural network for driver fatigue evaluation," IEEE transactions on neural networks and learning systems, 2019.

[100] K. Yue and D. Wang, "Eeg-based 3d visual fatigue evaluation using cnn," Electronics, vol. 8, no. 11, p. 1208, 2019.

[101] D. Shon, K. Im, J.-H. Park, D.-S. Lim, B. Jang, and J.-M. Kim, "Emotional stress state detection using genetic algorithm-based feature selection on eeg signals," International journal of environmental research and public health, vol. 15, no. 11, p. 2461, 2018. 
[102] H. Jebelli, M. M. Khalili, and S. Lee, "Mobile eeg-based workers' stress recognition by applying deep neural network," in Advances in Informatics and Computing in Civil and Construction Engineering. Springer, 2019, pp. 173-180.

[103] A. Sors, S. Bonnet, S. Mirek, L. Vercueil, and J.-F. Payen, "A convolutional neural network for sleep stage scoring from raw single-channel eeg," Biomedical Signal Processing and Control, vol. 42, pp. 107-114, 2018.

[104] M. M. Rahman, M. I. H. Bhuiyan, and A. R. Hassan, "Sleep stage classification using single-channel eog," Computers in biology and medicine, vol. 102, pp. 211-220, 2018.

[105] H. Phan, F. Andreotti, N. Cooray, O. Y. Chén, and M. De Vos, "Joint classification and prediction cnn framework for automatic sleep stage classification," IEEE Transactions on Biomedical Engineering, vol. 66, no. 5, pp. 1285-1296, 2018.

[106] Y. R. Tabar and U. Halici, "A novel deep learning approach for classification of eeg motor imagery signals," Journal of neural engineering, vol. 14, no. 1, p. 016003, 2016.

[107] H. Ramoser, J. Muller-Gerking, and G. Pfurtscheller, "Optimal spatial filtering of single trial eeg during imagined hand movement," IEEE transactions on rehabilitation engineering, vol. 8, no. 4, pp. 441-446, 2000.

[108] N. Korhan, Z. Dokur, and T. Olmez, "Motor imagery based eeg classification by using common spatial patterns and convolutional neural networks," in 2019 Scientific Meeting on ElectricalElectronics \& Biomedical Engineering and Computer Science (EBBT). IEEE, 2019, pp. 1-4.

[109] S. Sakhavi, C. Guan, and S. Yan, "Learning temporal information for brain-computer interface using convolutional neural networks," IEEE transactions on neural networks and learning systems, vol. 29, no. 11, pp. 5619-5629, 2018.

[110] B. E. Olivas-Padilla and M. I. Chacon-Murguia, "Classification of multiple motor imagery using deep convolutional neural networks and spatial filters," Applied Soft Computing, vol. 75, pp. 461-472, 2019.

[111] B. Ko, "A brief review of facial emotion recognition based on visual information," sensors, vol. 18, no. 2, p. 401, 2018.

[112] K.-Y. Wang, Y.-L. Ho, Y.-D. Huang, and W.-C. Fang, “Design of intelligent eeg system for human emotion recognition with convolutional neural network," in 2019 IEEE International Conference on Artificial Intelligence Circuits and Systems (AICAS). IEEE, 2019, pp. $142-145$

[113] Y. Yang, Q. Wu, M. Qiu, Y. Wang, and X. Chen, "Emotion recognition from multi-channel eeg through parallel convolutional recurrent neural network," in 2018 International Joint Conference on Neural Networks (IJCNN). IEEE, 2018, pp. 1-7.

[114] S. Lee, S. Han, and S. C. Jun, "Eeg hyperscanning for eight or more persons-feasibility study for emotion recognition using deep learning technique," in 2018 Asia-Pacific Signal and Information Processing Association Annual Summit and Conference (APSIPA ASC). IEEE, 2018, pp. 488-492.

[115] E. L. Broek, "Ubiquitous emotion-aware computing," Personal and Ubiquitous Computing, vol. 17, no. 1, pp. 53-67, 2013.

[116] Y.-P. Lin, C.-H. Wang, T.-P. Jung, T.-L. Wu, S.-K. Jeng, J.-R. Duann, and J.-H. Chen, "Eeg-based emotion recognition in music listening," IEEE Transactions on Biomedical Engineering, vol. 57, no. 7, pp. 1798-1806, 2010.

[117] S.-E. Moon, S. Jang, and J.-S. Lee, “Convolutional neural network approach for eeg-based emotion recognition using brain connectivity and its spatial information," in 2018 IEEE International Conference on Acoustics, Speech and Signal Processing (ICASSP). IEEE, 2018, pp. 2556-2560.

[118] S. Koelstra, C. Muhl, M. Soleymani, J.-S. Lee, A. Yazdani, T. Ebrahimi, T. Pun, A. Nijholt, and I. Patras, "Deap: A database for emotion analysis; using physiological signals," IEEE transactions on affective computing, vol. 3, no. 1, pp. 18-31, 2011.

[119] T. Song, W. Zheng, P. Song, and Z. Cui, "Eeg emotion recognition using dynamical graph convolutional neural networks," IEEE Transactions on Affective Computing, 2018.

[120] W.-L. Zheng and B.-L. Lu, "Investigating critical frequency bands and channels for eeg-based emotion recognition with deep neural networks," IEEE Transactions on Autonomous Mental Development, vol. 7, no. 3, pp. 162-175, 2015.

[121] S. Katsigiannis and N. Ramzan, "Dreamer: A database for emotion recognition through eeg and ecg signals from wireless lowcost off-the-shelf devices," IEEE journal of biomedical and health informatics, vol. 22, no. 1, pp. 98-107, 2017.
[122] K. G. Hartmann, R. T. Schirrmeister, and T. Ball, "Eeg-gan: Generative adversarial networks for electroencephalograhic (eeg) brain signals," arXiv preprint arXiv:1806.01875, 2018.

[123] S. M. Abdelfattah, G. M. Abdelrahman, and M. Wang, "Augmenting the size of eeg datasets using generative adversarial networks," in 2018 International Joint Conference on Neural Networks (IJCNN). IEEE, 2018, pp. 1-6.

[124] S. Panwar, P. Rad, T.-P. Jung, and Y. Huang, "Modeling eeg data distribution with a wasserstein generative adversarial network to predict rsvp events," arXiv preprint arXiv:1911.04379, 2019.

[125] Y. Luo and B.-L. Lu, "Eeg data augmentation for emotion recognition using a conditional wasserstein gan," in 2018 40th Annual International Conference of the IEEE Engineering in Medicine and Biology Society (EMBC). IEEE, 2018, pp. 2535-2538.

[126] Y. Luo, L.-Z. Zhu, and B.-L. Lu, "A gan-based data augmentation method for multimodal emotion recognition," in International Symposium on Neural Networks. Springer, 2019, pp. 141-150.

[127] I. A. Corley and Y. Huang, "Deep eeg super-resolution: Upsampling eeg spatial resolution with generative adversarial networks," in 2018 IEEE EMBS International Conference on Biomedical $\mathcal{E}$ Health Informatics (BHI). IEEE, 2018, pp. 100-103.

[128] F. Fahimi, Z. Zhang, W. B. Goh, K. K. Ang, and C. Guan, "Towards eeg generation using gans for bci applications," in 2019 IEEE EMBS International Conference on Biomedical \& Health Informatics (BHI). IEEE, 2019, pp. 1-4.

[129] S. Patnaik, L. Moharkar, and A. Chaudhari, "Deep rnn learning for eeg based functional brain state inference," in 2017 International Conference on Advances in Computing, Communication and Control (ICAC3). IEEE, 2017, pp. 1-6.

[130] C. Tan, F. Sun, W. Zhang, J. Chen, and C. Liu, "Multimodal classification with deep convolutional-recurrent neural networks for electroencephalography," in International Conference on Neural Information Processing. Springer, 2017, pp. 767-776.

[131] J. An and S. Cho, "Hand motion identification of grasp-andlift task from electroencephalography recordings using recurrent neural networks," in 2016 International Conference on Big Data and Smart Computing (BigComp). IEEE, 2016, pp. 427-429.

[132] S. Biswal, J. Kulas, H. Sun, B. Goparaju, M. B. Westover, M. T. Bianchi, and J. Sun, "Sleepnet: automated sleep staging system via deep learning," arXiv preprint arXiv:1707.08262, 2017.

[133] T. Zhang, W. Zheng, Z. Cui, Y. Zong, and Y. Li, "Spatial-temporal recurrent neural network for emotion recognition," IEEE transactions on cybernetics, vol. 49, no. 3, pp. 839-847, 2018.

[134] M. Attia, I. Hettiarachchi, M. Hossny, and S. Nahavandi, "A time domain classification of steady-state visual evoked potentials using deep recurrent-convolutional neural networks," in 2018 IEEE 15th International Symposium on Biomedical Imaging (ISBI 2018). IEEE, 2018, pp. 766-769.

[135] M.-A. Moinnereau, T. Brienne, S. Brodeur, J. Rouat, K. Whittingstall, and E. Plourde, "Classification of auditory stimuli from eeg signals with a regulated recurrent neural network reservoir," arXiv preprint arXiv:1804.10322, 2018.

[136] C. Spampinato, S. Palazzo, I. Kavasidis, D. Giordano, N. Souly, and M. Shah, "Deep learning human mind for automated visual classification," in Proceedings of the IEEE Conference on Computer Vision and Pattern Recognition, 2017, pp. 6809-6817.

[137] A. Supratak, H. Dong, C. Wu, and Y. Guo, "Deepsleepnet: A model for automatic sleep stage scoring based on raw singlechannel eeg," IEEE Transactions on Neural Systems and Rehabilitation Engineering, vol. 25, no. 11, pp. 1998-2008, 2017.

[138] M. M. Hasib, T. Nayak, and Y. Huang, "A hierarchical lstm model with attention for modeling eeg non-stationarity for human decision prediction," in 2018 IEEE EMBS international conference on biomedical \& health informatics (BHI). IEEE, 2018, pp. 104-107.

[139] H. Dong, A. Supratak, W. Pan, C. Wu, P. M. Matthews, and Y. Guo, "Mixed neural network approach for temporal sleep stage classification," IEEE Transactions on Neural Systems and Rehabilitation Engineering, vol. 26, no. 2, pp. 324-333, 2017.

[140] S. Palazzo, C. Spampinato, I. Kavasidis, D. Giordano, and M. Shah, "Generative adversarial networks conditioned by brain signals," in Proceedings of the IEEE International Conference on Computer Vision, 2017, pp. 3410-3418.

[141] G. Krishna, Y. Han, C. Tran, M. Carnahan, and A. H. Tewfik, "State-of-the-art speech recognition using eeg and towards decoding of speech spectrum from eeg," arXiv preprint arXiv:1908.05743, 2019. 
[142] C. Tan, F. Sun, T. Kong, W. Zhang, C. Yang, and C. Liu, "A survey on deep transfer learning," in International Conference on Artificial Neural Networks. Springer, 2018, pp. 270-279.

[143] M. Mahmud, M. S. Kaiser, A. Hussain, and S. Vassanelli, “Applications of deep learning and reinforcement learning to biological data," IEEE transactions on neural networks and learning systems, vol. 29, no. 6, pp. 2063-2079, 2018.

[144] S. A. Prajapati, R. Nagaraj, and S. Mitra, "Classification of dental diseases using cnn and transfer learning," in 2017 5th International Symposium on Computational and Business Intelligence (ISCBI). IEEE, 2017, pp. 70-74.

[145] G. Wimmer, A. Vécsei, and A. Uhl, “Cnn transfer learning for the automated diagnosis of celiac disease," in 2016 Sixth International Conference on Image Processing Theory, Tools and Applications (IPTA). IEEE, 2016, pp. 1-6.

[146] A. Khatami, M. Babaie, H. R. Tizhoosh, A. Khosravi, T. Nguyen and S. Nahavandi, "A sequential search-space shrinking using cnn transfer learning and a radon projection pool for medical image retrieval," Expert Systems with Applications, vol. 100, pp. 224-233, 2018

[147] D. Han, Q. Liu, and W. Fan, "A new image classification method using cnn transfer learning and web data augmentation," Expert Systems with Applications, vol. 95, pp. 43-56, 2018.

[148] L. A. Alexandre, "3d object recognition using convolutional neural networks with transfer learning between input channels," in Intelligent Autonomous Systems 13. Springer, 2016, pp. 889-898.

[149] S. Sakhavi and C. Guan, "Convolutional neural network-based transfer learning and knowledge distillation using multi-subject data in motor imagery bci," in 2017 8th International IEEE/EMBS Conference on Neural Engineering (NER). IEEE, 2017, pp. 588-591.

[150] G. Xu, X. Shen, S. Chen, Y. Zong, C. Zhang, H. Yue, M. Liu, F. Chen, and W. Che, "A deep transfer convolutional neural network framework for eeg signal classification," IEEE Access, vol. 7, pp. 112 767-112776, 2019.

[151] H. Dose, J. S. Møller, H. K. Iversen, and S. Puthusserypady, "An end-to-end deep learning approach to mi-eeg signal classification for bcis," Expert Systems with Applications, vol. 114, pp. 532-542, 2018.

[152] X. Zhu, P. Li, C. Li, D. Yao, R. Zhang, and P. Xu, "Separated channel convolutional neural network to realize the training free motor imagery bci systems," Biomedical Signal Processing and Control, vol. 49, pp. 396-403, 2019.

[153] C. Szegedy, W. Zaremba, I. Sutskever, J. Bruna, D. Erhan, I. Goodfellow, and R. Fergus, "Intriguing properties of neural networks," arXiv preprint arXiv:1312.6199, 2013.

[154] I. J. Goodfellow, J. Shlens, and C. Szegedy, "Explaining and harnessing adversarial examples," stat, vol. 1050, p. 20, 2015.

[155] A. Kurakin, I. Goodfellow, and S. Bengio, "Adversarial examples in the physical world," arXiv preprint arXiv:1607.02533, 2016.

[156] A. Athalye, L. Engstrom, A. Ilyas, and K. Kwok, "Synthesizing robust adversarial examples," arXiv preprint arXiv:1707.07397, 2017.

[157] J. Li, K. Cheng, S. Wang, F. Morstatter, R. P. Trevino, J. Tang, and H. Liu, "Feature selection: A data perspective," ACM Computing Surveys (CSUR), vol. 50, no. 6, p. 94, 2018.

[158] X. Zhang and D. Wu, "On the vulnerability of cnn classifiers in eeg-based bcis," IEEE Transactions on Neural Systems and Rehabilitation Engineering, vol. 27, no. 5, pp. 814-825, 2019.

[159] V. J. Lawhern, A. J. Solon, N. R. Waytowich, S. M. Gordon, C. P. Hung, and B. J. Lance, "Eegnet: a compact convolutional neural network for eeg-based brain-computer interfaces," Journal of neural engineering, vol. 15, no. 5, p. 056013, 2018.

[160] R. T. Schirrmeister, J. T. Springenberg, L. D. J. Fiederer, M. Glasstetter, K. Eggensperger, M. Tangermann, F. Hutter, W. Burgard, and T. Ball, "Deep learning with convolutional neural networks for eeg decoding and visualization," Human brain mapping, vol. 38, no. 11, pp. 5391-5420, 2017.

[161] X. Jiang, X. Zhang, and D. Wu, "Active learning for black-box adversarial attacks in eeg-based brain-computer interfaces," in 2019 IEEE Symposium Series on Computational Intelligence (SSCI), 2019, pp. 361-368.

[162] L. Meng, C.-T. Lin, T.-P. Jung, and D. Wu, "White-box target attacks for eeg-based bci regression problems," in International Conference on Neural Information Processing, Sydney, Australia, December 2019.
[163] Z. Liu, X. Zhang, and D. Wu, "Universal adversarial perturbations for cnn classifiers in eeg-based bcis," arXiv preprint arXiv:1912.01171, 2019.

[164] T. Miller, "” but why?" understanding explainable artificial intelligence," XRDS: Crossroads, The ACM Magazine for Students, vol. 25, no. 3, pp. 20-25, 2019.

[165] K. Chen, L. Yao, D. Zhang, X. Chang, G. Long, and S. Wang, "Distributionally robust semi-supervised learning for peoplecentric sensing," in Proceedings of the AAAI Conference on Artificial Intelligence, vol. 33, 2019, pp. 3321-3328.

[166] R. Donovan and X.-H. Yu, "Motor imagery classification using tsk fuzzy inference neural networks," in 2018 International Joint Conference on Neural Networks (IJCNN). IEEE, 2018, pp. 1-6.

[167] Y. Sun, F. P.-W. Lo, and B. Lo, "Eeg-based user identification system using $1 \mathrm{~d}$-convolutional long short-term memory neural networks," Expert Systems with Applications, vol. 125, pp. 259-267, 2019.

[168] W. Chen, S. Wang, X. Zhang, L. Yao, L. Yue, B. Qian, and X. Li, "Eeg-based motion intention recognition via multi-task rnns," in Proceedings of the 2018 SIAM International Conference on Data Mining. SIAM, 2018, pp. 279-287.

[169] D. Zhang, L. Yao, K. Chen, S. Wang, X. Chang, and Y. Liu, "Making sense of spatio-temporal preserving representations for eeg-based human intention recognition," IEEE transactions on cybernetics, 2019.

[170] Q. Zheng, F. Zhu, J. Qin, and P.-A. Heng, "Multiclass support matrix machine for single trial eeg classification," Neurocomputing, vol. 275, pp. 869-880, 2018.

[171] A. K. Das, S. Sundaram, and N. Sundararajan, "A self-regulated interval type-2 neuro-fuzzy inference system for handling nonstationarities in eeg signals for bci," IEEE Transactions on Fuzzy Systems, vol. 24, no. 6, pp. 1565-1577, 2016.

[172] T.-j. Luo, F. Chao et al., "Exploring spatial-frequency-sequential relationships for motor imagery classification with recurrent neural network," BMC bioinformatics, vol. 19, no. 1, p. 344, 2018.

[173] K. Yan, L. Kou, and D. Zhang, "Learning domain-invariant subspace using domain features and independence maximization," IEEE transactions on cybernetics, vol. 48, no. 1, pp. 288-299, 2017.

[174] Z. Lan, O. Sourina, L. Wang, R. Scherer, and G. R. MüllerPutz, "Domain adaptation techniques for eeg-based emotion recognition: a comparative study on two public datasets," IEEE Transactions on Cognitive and Developmental Systems, vol. 11, no. 1, pp. 85-94, 2018.

[175] A. Chatchinarat, K. Wong, and C. Fung, "Fuzzy classification of human emotions using fuzzy c-mean ( $\mathrm{fcfcm}), "$ in 2016 International Conference on Fuzzy Theory and Its Applications (iFuzzy). IEEE, 2016, pp. 1-5.

[176] Y. Luo, S.-Y. Zhang, W.-L. Zheng, and B.-L. Lu, "Wgan domain adaptation for eeg-based emotion recognition," in International Conference on Neural Information Processing. Springer, 2018, pp. 275-286.

[177] S. Alhagry, A. A. Fahmy, and R. A. El-Khoribi, "Emotion recognition based on eeg using lstm recurrent neural network," Emotion, vol. 8, no. 10, pp. 355-358, 2017.

[178] X. Chai, Q. Wang, Y. Zhao, X. Liu, O. Bai, and Y. Li, “Unsupervised domain adaptation techniques based on auto-encoder for non-stationary eeg-based emotion recognition," Computers in biology and medicine, vol. 79, pp. 205-214, 2016.

[179] P. Bashivan, I. Rish, and S. Heisig, "Mental state recognition via wearable eeg," arXiv preprint arXiv:1602.00985, 2016.

[180] J. R. Brazète, J.-F. Gagnon, R. B. Postuma, J.-A. Bertrand, D. Petit, and J. Montplaisir, "Electroencephalogram slowing predicts neurodegeneration in rapid eye movement sleep behavior disorder," Neurobiology of aging, vol. 37, pp. 74-81, 2016.

[181] S. S. Talathi, "Deep recurrent neural networks for seizure detection and early seizure detection systems," arXiv preprint arXiv:1706.03283, 2017.

[182] K. M. Tsiouris, V. C. Pezoulas, M. Zervakis, S. Konitsiotis, D. D. Koutsouris, and D. I. Fotiadis, "A long short-term memory deep learning network for the prediction of epileptic seizures using eeg signals," Computers in biology and medicine, vol. 99, pp. 24-37, 2018

[183] A. Gupta, P. Singh, and M. Karlekar, "A novel signal modeling approach for classification of seizure and seizure-free eeg signals," IEEE Transactions on Neural Systems and Rehabilitation Engineering, vol. 26, no. 5, pp. 925-935, 2018. 
[184] V. Shah, M. Golmohammadi, S. Ziyabari, E. Von Weltin, I. Obeid, and J. Picone, "Optimizing channel selection for seizure detection," in 2017 IEEE Signal Processing in Medicine and Biology Symposium (SPMB). IEEE, 2017, pp. 1-5.

[185] R. Alkawadri, "Brain computer interface (bci) applications in mapping of epileptic brain networks based on intracranial-eeg," Frontiers in Neuroscience, vol. 13, p. 191, 2019.

[186] S. L. Oh, Y. Hagiwara, U. Raghavendra, R. Yuvaraj, N. Arunkumar, M. Murugappan, and U. R. Acharya, "A deep learning approach for parkinson's disease diagnosis from eeg signals," Neural Computing and Applications, pp. 1-7, 2018.

[187] G. Ruffini, D. Ibañez, M. Castellano, S. Dunne, and A. SoriaFrisch, "Eeg-driven rnn classification for prognosis of neurodegeneration in at-risk patients," in International Conference on Artificial Neural Networks. Springer, 2016, pp. 306-313.

[188] F. C. Morabito, M. Campolo, C. Ieracitano, J. M. Ebadi, L. Bonanno, A. Bramanti, S. Desalvo, N. Mammone, and P. Bramanti, "Deep convolutional neural networks for classification of mild cognitive impaired and alzheimer's disease patients from scalp eeg recordings," in 2016 IEEE 2nd International Forum on Research and Technologies for Society and Industry Leveraging a better tomorrow (RTSI). IEEE, 2016, pp. 1-6.

[189] S. Simpraga, R. Alvarez-Jimenez, H. D. Mansvelder, J. M. Van Gerven, G. J. Groeneveld, S.-S. Poil, and K. LinkenkaerHansen, "Eeg machine learning for accurate detection of cholinergic intervention and alzheimer's disease," Scientific reports, vol. 7, no. 1, p. 5775, 2017.

[190] M. Shim, H.-J. Hwang, D.-W. Kim, S.-H. Lee, and C.-H. Im, "Machine-learning-based diagnosis of schizophrenia using combined sensor-level and source-level eeg features," Schizophrenia research, vol. 176, no. 2-3, pp. 314-319, 2016.

[191] L. Chu, R. Qiu, H. Liu, Z. Ling, T. Zhang, and J. Wang, “Individual recognition in schizophrenia using deep learning methods with random forest and voting classifiers: Insights from resting state eeg streams," arXiv preprint arXiv:1707.03467, 2017.

[192] S. R. Soekadar, N. Birbaumer, M. W. Slutzky, and L. G. Cohen, "Brain-machine interfaces in neurorehabilitation of stroke," Neurobiology of disease, vol. 83, pp. 172-179, 2015.

[193] A. Remsik, B. Young, R. Vermilyea, L. Kiekhoefer, J. Abrams, S. Evander Elmore, P. Schultz, V. Nair, D. Edwards, J. Williams et al., "A review of the progression and future implications of brain-computer interface therapies for restoration of distal upper extremity motor function after stroke," Expert review of medical devices, vol. 13, no. 5, pp. 445-454, 2016.

[194] A. A. Frolov, O. Mokienko, R. Lyukmanov, E. Biryukova, S. Kotov, L. Turbina, G. Nadareyshvily, and Y. Bushkova, "Post-stroke rehabilitation training with a motor-imagery-based brain-computer interface (bci)-controlled hand exoskeleton: a randomized controlled multicenter trial," Frontiers in neuroscience, vol. 11, p. 400, 2017.

[195] E. Clark, A. Czaplewski, S. Dourney, A. Gadelha, K. Nguyen, P. Pasciucco, M. Rios, R. Stuart, E. Castillo, and M. Korostenskaja, "Brain-computer interface for motor rehabilitation," in International Conference on Human-Computer Interaction. Springer, 2019, pp. 243-254.

[196] M. A. Cervera, S. R. Soekadar, J. Ushiba, J. d. R. Millán, M. Liu, N. Birbaumer, and G. Garipelli, "Brain-computer interfaces for post-stroke motor rehabilitation: a meta-analysis," Annals of clinical and translational neurology, vol. 5, no. 5, pp. 651-663, 2018.

[197] D. C. Irimia, W. Cho, R. Ortner, B. Z. Allison, B. E. Ignat, G. Edlinger, and C. Guger, "Brain-computer interfaces with multi-sensory feedback for stroke rehabilitation: a case study," Artificial organs, vol. 41, no. 11, pp. E178-E184, 2017.

[198] Z.-H. Cao, L.-W. Ko, K.-L. Lai, S.-B. Huang, S.-J. Wang, and C.-T. Lin, "Classification of migraine stages based on resting-state eeg power," in 2015 International Joint Conference on Neural Networks (IJCNN). IEEE, 2015, pp. 1-5.

[199] Z. Cao, C.-T. Lin, C.-H. Chuang, K.-L. Lai, A. C. Yang, J.-L. Fuh, and S.-J. Wang, "Resting-state eeg power and coherence vary between migraine phases," The journal of headache and pain, vol. 17, no. 1, p. 102, 2016.

[200] Z. Cao, K.-L. Lai, C.-T. Lin, C.-H. Chuang, C.-C. Chou, and S.-J. Wang, "Exploring resting-state eeg complexity before migraine attacks," Cephalalgia, vol. 38, no. 7, pp. 1296-1306, 2018.

[201] C.-T. Lin, C.-H. Chuang, Z. Cao, A. K. Singh, C.-S. Hung, Y.-H. Yu, M. Nascimben, Y.-T. Liu, J.-T. King, T.-P. Su et al., "Forehead eeg in support of future feasible personal healthcare solutions: sleep management, headache prevention, and depression treatment," IEEE Access, vol. 5, pp. 10612-10621, 2017.

[202] Z. Cao, C.-T. Lin, W. Ding, M.-H. Chen, C.-T. Li, and T.-P. $\mathrm{Su}$, "Identifying ketamine responses in treatment-resistant depression using a wearable forehead eeg," IEEE Transactions on Biomedical Engineering, vol. 66, no. 6, pp. 1668-1679, 2018.

[203] A. U. Patil, A. Dube, R. K. Jain, G. D. Jindal, and D. Madathil, "Classification and comparative analysis of control and migraine subjects using eeg signals," in Information Systems Design and Intelligent Applications. Springer, 2019, pp. 31-39.

[204] Z. Cao, M. Prasad, and C.-T. Lin, "Estimation of ssvep-based eeg complexity using inherent fuzzy entropy," in 2017 IEEE International Conference on Fuzzy Systems (FUZZ-IEEE). IEEE, 2017, pp. 1-5.

[205] D. Camfferman, G. L. Moseley, K. Gertz, M. W. Pettet, and M. P. Jensen, "Waking eeg cortical markers of chronic pain and sleepiness," Pain Medicine, vol. 18, no. 10, pp. 1921-1931, 2017.

[206] T. Yanagisawa, R. Fukuma, B. Seymour, K. Hosomi, H. Kishima, T. Shimizu, H. Yokoi, M. Hirata, T. Yoshimine, Y. Kamitani et al., "Using a bci prosthetic hand to control phantom limb pain," in Brain-Computer Interface Research. Springer, 2019, pp. 43-52.

[207] U. R. Acharya, S. L. Oh, Y. Hagiwara, J. H. Tan, H. Adeli, and D. P. Subha, "Automated eeg-based screening of depression using deep convolutional neural network," Computer methods and programs in biomedicine, vol. 161, pp. 103-113, 2018.

[208] X. Li, R. La, Y. Wang, J. Niu, S. Zeng, S. Sun, and J. Zhu, "Eegbased mild depression recognition using convolutional neural network," Medical \& biological engineering \& computing, vol. 57, no. 6, pp. 1341-1352, 2019.

[209] S.-C. Liao, C.-T. Wu, H.-C. Huang, W.-T. Cheng, and Y.-H. Liu, "Major depression detection from eeg signals using kernel eigenfilter-bank common spatial patterns," Sensors, vol. 17, no. 6, p 1385, 2017.

[210] P. Fiedler, R. Mühle, S. Griebel, P. Pedrosa, C. Fonseca, F. Vaz, F. Zanow, and J. Haueisen, "Contact pressure and flexibility of multipin dry eeg electrodes," IEEE Transactions on Neural Systems and Rehabilitation Engineering, vol. 26, no. 4, pp. 750-757, 2018.

[211] C.-T. Lin, Y.-H. Yu, J.-T. King, C.-H. Liu, and L.-D. Liao, "Augmented wire-embedded silicon-based dry-contact sensors for electroencephalography signal measurements," IEEE Sensors Journal, 2019.

[212] B. Houston, M. Thompson, A. Ko, and H. Chizeck, "A machinelearning approach to volitional control of a closed-loop deep brain stimulation system," Journal of neural engineering, vol. 16, no. 1, p. 016004, 2018.

[213] J. Liu, S. Qu, W. Chen, J. Chu, and Y. Sun, "Online adaptive decoding of motor imagery based on reinforcement learning," in 2019 14th IEEE Conference on Industrial Electronics and Applications (ICIEA). IEEE, 2019, pp. 522-527.

[214] G. Müller-Putz, R. Leeb, M. Tangermann, J. Höhne, A. Kübler, F. Cincotti, D. Mattia, R. Rupp, K.-R. Müller, and J. d. R. Millán, "Towards noninvasive hybrid brain-computer interfaces: framework, practice, clinical application, and beyond," Proceedings of the IEEE, vol. 103, no. 6, pp. 926-943, 2015.

[215] K.-S. Hong and M. J. Khan, "Hybrid brain-computer interface techniques for improved classification accuracy and increased number of commands: a review," Frontiers in neurorobotics, vol. 11, p. 35, 2017.

[216] U. H. Govindarajan, A. J. Trappey, and C. V. Trappey, "Immersive technology for human-centric cyberphysical systems in complex manufacturing processes: a comprehensive overview of the global patent profile using collective intelligence," Complexity, vol. 2018, 2018.

[217] L. Angrisani, P. Arpaia, N. Moccaldi, and A. Esposito, "Wearable augmented reality and brain computer interface to improve human-robot interactions in smart industry: A feasibility study for ssvep signals," in 2018 IEEE 4th International Forum on Research and Technology for Society and Industry (RTSI). IEEE, 2018, pp. 1-5.

[218] R. Zerafa, T. Camilleri, O. Falzon, and K. P. Camilleri, "A realtime ssvep-based brain-computer interface music player application," in XIV Mediterranean Conference on Medical and Biological Engineering and Computing 2016. Springer, 2016, pp. 173-178.

[219] J. Faller, B. Z. Allison, C. Brunner, R. Scherer, D. Schmalstieg, G. Pfurtscheller, and C. Neuper, "A feasibility study on ssvepbased interaction with motivating and immersive virtual and augmented reality," arXiv preprint arXiv:1701.03981, 2017. 